Discrete Comput Geom 36:331-361 (2006)

DOI: $10.1007 / \mathrm{s} 00454-006-1243-1$

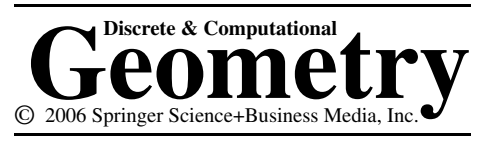

\title{
A Geometric Study of the Split Decomposition
}

\author{
Hiroshi Hirai \\ Research Institute for Mathematical Sciences, Kyoto University, \\ Kyoto, 606-8502, Japan \\ hirai@kurims.kyoto-u.ac.jp
}

\begin{abstract}
This paper sheds new light on split decomposition theory and T-theory from the viewpoint of convex analysis and polyhedral geometry. By regarding finite metrics as discrete concave functions, Bandelt-Dress' split decomposition can be derived as a special case of more general decomposition of polyhedral/discrete concave functions introduced in this paper. It is shown that the combinatorics of splits discussed in connection with the split decomposition corresponds to the geometric properties of a hyperplane arrangement and a point configuration. Using our approach, the split decomposition of metrics can be naturally extended to distance functions, which may violate the triangle inequality, using partial split distances.
\end{abstract}

\section{Introduction}

Mathematical treatment of metric spaces on a finite set (finite metric spaces) has come to be increasingly important in the area of bioinfomatics and phylogenetics; see [2] and [24]. The central problem in phylogenetics is reconstructing phylogenetic trees from given experimental data, e.g., DNA sequences. If the data is given as a distance matrix expressing dissimilarity between species, the problem is to search for a tree metric that "fits" the given distance matrix, where a metric is called a tree metric if it can be represented as the path metric on some weighted tree.

T-theory [9], developed by Dress and coworkers, provides a beautiful mathematical framework for this phylogenetic problem. The split decomposition, due to Bandelt and Dress [1], is a T-theoretical phylogenetic analysis method, which is briefly summarized as follows; see Section 4 for precise definitions. Let $V$ be a finite set and let $d: V \times V \rightarrow \mathbf{R}$ be a metric on $V$. Then the split decomposition decomposes $d$ into the following form:

$$
d=\sum_{\{A, B\}: \text { split }} \alpha_{\{A, B\}}^{d} \delta_{\{A, B\}}+d^{\prime}
$$




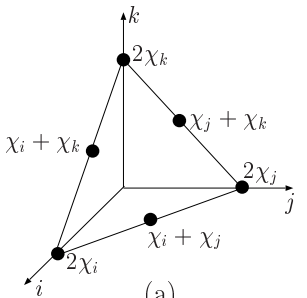

(a)

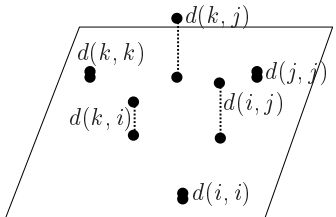

(b)

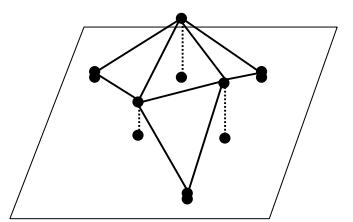

(c)

Fig. 1. Three-dimensional example.

where a split means a bipartition of $V, \delta_{\{A, B\}}$ is the split metric defined in (4.3), $\alpha_{\{A, B\}}^{d}$ is the nonnegative number called the isolation index defined in (4.4), and $d^{\prime}$ is a split-prime metric.

One of the main aims of this paper is to derive the split decomposition in a natural way as a special case of a decomposition of (discrete) convex/concave functions that we propose in this paper. This viewpoint of convex analysis sheds new light on such important concepts as split decomposition, tight span, isolation index, and weakly compatible splits. Furthermore, our approach leads to a natural extension of the split decomposition for distances, which may violate the triangle inequality.

To describe our key idea to understand the split decomposition from the viewpoint of polyhedral geometry, let us take the following small example. Let $V=\{i, j, k\}$ be a 3-point set and let $d: V \times V \rightarrow \mathbf{R}$ be a metric on $V$ defined as

$$
d=\begin{array}{|l|lll|}
\hline & i & j & k \\
\hline i & 0 & 4 & 2 \\
j & 4 & 0 & 4 \\
k & 2 & 4 & 0 \\
\hline
\end{array}
$$

Consider the point set $\left\{\left(\chi_{i}+\chi_{j}, d(i, j)\right) \mid i, j \in V\right\} \subseteq \mathbf{R}^{V} \times \mathbf{R}$ and the upper envelope of its convex hull, where $\chi_{i}$ is the $i$ th unit vector. Namely, we regard $d$ as a function defined on the point set $\Lambda=\left\{\chi_{i}+\chi_{j} \mid i, j \in V\right\}$. We illustrate this situation in Fig. 1. The point set $\Lambda$ consists of vertices of the simplex and midpoints of its edges, and lies on the hyperplane $\left\{x \in \mathbf{R}^{V} \mid \sum_{i \in V} x(i)=2\right\}$ (Fig. 1(a)). Hence, we can project $\left\{\left(\chi_{i}+\chi_{j}, d(i, j)\right) \mid i, j \in V\right\}$ to three-dimensional space (Fig. 1(b)).

We observe that the concave function of this upper envelope can be decomposed into a sum of three simple concave functions as in Fig. 2. Then this decomposition of the

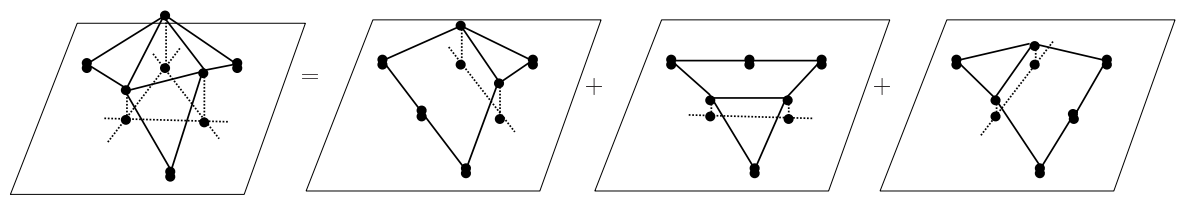

Fig. 2. Decomposition of the concave function associated with $d$. 
concave function associated with $d$ induces the decomposition of $d$ as

$$
\begin{aligned}
d & =2\left(\begin{array}{lll}
0 & 1 & 0 \\
1 & 0 & 1 \\
0 & 1 & 0
\end{array}\right)+\left(\begin{array}{lll}
0 & 1 & 1 \\
1 & 0 & 0 \\
1 & 0 & 0
\end{array}\right)+\left(\begin{array}{lll}
0 & 0 & 1 \\
0 & 0 & 1 \\
1 & 1 & 0
\end{array}\right) \\
& =2 \delta_{\{2\},\{1,3\}}+\delta_{\{1\},\{2,3\}}+\delta_{\{3\},\{1,2\}} .
\end{aligned}
$$

As will be shown in this paper, this decomposition coincides with Bandelt-Dress' split decomposition.

The observation above motivates us to study the following type of a decomposition of a polyhedral convex function $f$ on $\mathbf{R}^{n}$ as

$$
f(x)=\sum_{(a, r) \in \mathbf{R}^{n} \times \mathbf{R}} c_{a, r}^{f}|\langle a, x\rangle-r|+f^{\prime}(x) \quad\left(x \in \mathbf{R}^{n}\right),
$$

where $c_{a, r}^{f}$ is the nonnegative number defined by $\sup \{t \geq 0|f-t|\langle a, x\rangle-r \mid$ is convex $\}$ and $f^{\prime}$ is a polyhedral convex function satisfying $c_{a, r}^{f^{\prime}} \in\{0,+\infty\}$ for all $(a, r) \in \mathbf{R}^{n} \times \mathbf{R}$. We call function $|\langle a, x\rangle-r|$ a split function corresponding to a split metric. Indeed, the terms of the right-hand side of Fig. 2 can be regarded as the negative of split functions. We show in Section 2 that this decomposition (1.4) is uniquely determined (Theorem 2.2). We call this the polyhedral split decomposition.

As with the above example, this decomposition yields the decomposition of a function defined on a finite point set $X$. In Section 3 we study the split decomposition for a function defined on $X$ (the split decomposition of discrete functions).

In Section 4 we regard a metric $d$ as a function on $\Lambda$ as in Fig. 1 and apply the results of Sections 2 and 3. We then obtain the following:

- By discretizing the polyhedral split decomposition (1.4), Bandelt-Dress' split decomposition (1.1) can be derived; see Proposition 4.5 and Theorems 4.6 and 4.7.

- The split decomposition of metrics can be naturally extended for distance functions, which may possibly violate the triangle inequality, using partial split distances; see Theorem 4.7 and equations (4.13) and (4.27). This extended split decomposition exploits more detailed combinatorial structure of a given distance matrix than Bandelt-Dress' split decomposition (1.1) in the case that $d$ violates the triangle inequality; see Remark 4.8. For example, a distance on subtrees on a tree defined by the shortest path length between subtrees (Fig. 3), studied by Hirai [15], is a totally split-decomposable distance in our sense, which can be regarded as an extension of the fact that a tree metric is a totally split-decomposable metric in Bandelt-Dress' sense; $d^{\prime}=0$ in (1.1). So such distances can be recognized by our extended split decomposition; see Remark 4.18.

- We show the split-hyperplane correspondence, where each split can be associated with a certain hyperplane dividing simplex and weak compatibility of splits can be translated into a certain geometric property of the corresponding hyperplane arrangement and the point configuration $\Lambda$; see Theorem 4.14 and Proposition 4.16.

Closing this Introduction, we mention some background of the present work. Applications of convex analysis to combinatorial and discrete structures are given in the 


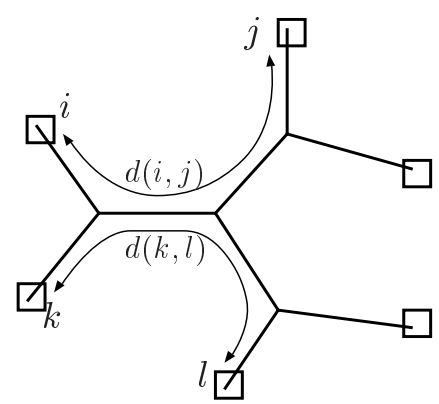

(a)

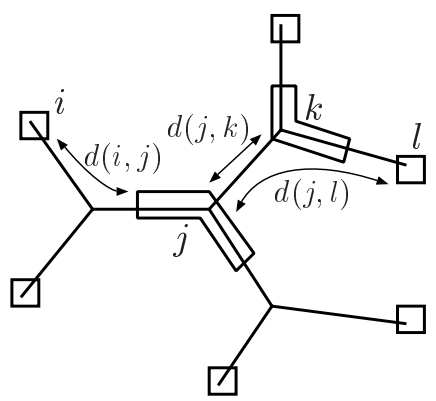

(b)

Fig. 3. (a) Tree metric and (b) distance between subtrees on a tree.

theory of submodular functions by Lovász [19], Frank [11], and Fujishige [12] (see also [13]). Recently Murota [20] developed discrete convex analysis, a convex analysis for functions defined on integer lattice points. Hirai and Murota [16] discuss the relationship between tree metrics and discrete convexity, where they observe that $d$ is a tree metric if and only if the function on $\Lambda$ associated with $d$, as in Fig. 1, is an M-concave function, which is one of the fundamental classes of discrete convex functions. The present paper is strongly influenced by these works.

\section{Split Decomposition of Polyhedral Convex Functions}

In this section we derive the polyhedral split decomposition (1.4), which is the basis for subsequent developments in this paper.

\subsection{Basic Notation}

Here we introduce some basic notation about convexity and polyhedra; see [22], [21], and [27]. Let $\mathbf{R}, \mathbf{R}_{+}$, and $\mathbf{R}_{++}$be the sets of real numbers, nonnegative real numbers, and positive real numbers, respectively. Let $\mathbf{R}^{n}$ be the $n$-dimensional Euclidean space with the standard inner product $\langle\cdot, \cdot\rangle$. The norm $\|x\|$ of $x \in \mathbf{R}^{n}$ is defined by $\sqrt{\langle x, x\rangle}$. For $x, y \in \mathbf{R}^{n}$, let $[x, y]$ denote the closed line segment between $x$ and $y$. For two subset $F$, $G \subseteq \mathbf{R}^{n}, F+G$ means the Minkowski sum of $F$ and $G$ defined by $\{x+y \mid x \in F, y \in G\}$. A subset $S \subseteq \mathbf{R}^{n}$ is said to be convex if $[x, y] \subseteq S$ for each $x, y \in \mathbf{R}$, and is said to be (convex) cone if $\alpha x+\beta y \in S$ for $x, y \in S$ and $\alpha, \beta \in \mathbf{R}_{+}$. For a subset $X \subseteq \mathbf{R}^{n}$, we denote by conv $X$, cone $X$, and aff $X$ the convex hull (the smallest convex set containing $X$ ), the conical hull (the smallest convex cone containing $X$ ), and the affine hull (the smallest affine space containing $X$ ), respectively, i.e.,

$$
\operatorname{conv} X=\left\{\sum_{y \in Y} \lambda_{y} y \mid Y \subseteq X \text { : finite set, } \lambda \in \mathbf{R}_{+}^{Y}, \sum_{y \in Y} \lambda_{y}=1\right\} \text {, }
$$




$$
\begin{aligned}
\text { cone } X & =\left\{\sum_{y \in Y} \lambda_{y} y \mid Y \subseteq X: \text { finite set, } \lambda \in \mathbf{R}_{+}^{Y}\right\}, \\
\text { aff } X & =\left\{\sum_{y \in Y} \lambda_{y} y \mid Y \subseteq X \text { : finite set, } \lambda \in \mathbf{R}^{Y}, \sum_{y \in Y} \lambda_{y}=0\right\} .
\end{aligned}
$$

int $X$ and ri $X$ mean the set of interior points and relative interior points of $X$, respectively, i.e., int $X=\left\{x \in X \mid \exists \varepsilon>0,\{x\}+B_{\varepsilon} \subseteq X\right\}$ and $\operatorname{ri} X=\{x \in X \mid \exists \varepsilon>0,\{x\}+$ aff $\left.X \cap B_{\varepsilon} \subseteq X\right\}$, where $B_{\varepsilon}=\{x \in \mathbf{R} \mid\|x\| \leq \varepsilon\}$. In particular, for $(a, r) \in \mathbf{R}^{n} \times \mathbf{R}$, we define a hyperplane $H_{a, r}$ by $\left\{x \in \mathbf{R}^{n} \mid\langle a, x\rangle=r\right\}$, closed half-spaces $H_{a, r}^{-}$and $H_{a, r}^{+}$ by $\left\{x \in \mathbf{R}^{n} \mid\langle a, x\rangle \leq r\right\}$ and $\left\{x \in \mathbf{R}^{n} \mid\langle a, x\rangle \geq r\right\}$, and open half-spaces $H_{a, r}^{--}$and $H_{a, r}^{++}$by $\left\{x \in \mathbf{R}^{n} \mid\langle a, x\rangle<r\right\}$ and $\left\{x \in \mathbf{R}^{n} \mid\langle a, x\rangle>r\right\}$. A subset $P \subseteq \mathbf{R}^{n}$ is a polyhedron if $P$ is the intersection of a finite number of closed half-spaces. A function $f: \mathbf{R}^{n} \rightarrow \mathbf{R} \cup\{+\infty\}$ is said to be convex if it satisfies

$$
\lambda f(x)+(1-\lambda) f(y) \geq f(\lambda x+(1-\lambda) y)
$$

for all $x, y \in \mathbf{R}^{n}, \lambda \in[0,1]$. dom $f=\left\{x \in \mathbf{R}^{n} \mid f(x)<+\infty\right\}$ is called the effective domain of $f$ and epi $f=\left\{(x, r) \in \mathbf{R}^{n} \times \mathbf{R} \mid f(x) \leq r\right\}$ is called the epigraph of $f$. The indicator function $\delta_{S}: \mathbf{R}^{n} \rightarrow \mathbf{R} \cup\{+\infty\}$ for a subset $S \subseteq \mathbf{R}^{n}$ is defined by

$$
\delta_{S}(x)=\left\{\begin{array}{ll}
0 & \text { if } x \in S \\
+\infty & \text { otherwise }
\end{array} \quad\left(x \in \mathbf{R}^{n}\right) .\right.
$$

A function $f$ is called a polyhedral convex function if its epigraph epi $f$ is polyhedral, or, equivalently, $f$ is represented by $\max \left\{\left\langle a_{i}, \cdot\right\rangle+b_{i} \mid 1 \leq i \leq m\right\}+\delta_{P}$ for $\left\{\left(a_{i}, b_{i}\right)\right\}_{i=1}^{m} \subseteq$ $\mathbf{R}^{n} \times \mathbf{R}$ and a polyhedron $P$.

A nonempty subset $F$ of a polyhedron $P$ is called a face of $P$ if $F=P$ or $F$ can be represented as $H \cap P$ for some hyperplane $H$ with $P \subseteq H^{+}$.

A polyhedral complex $\mathcal{C}$ is a finite collection of polyhedra such that (1) if $P \in \mathcal{C}$, all the faces of $P$ are also in $\mathcal{C}$, and (2) the nonempty intersection $P \cap Q$ of two polyhedra $P, Q \in \mathcal{C}$ is a face of $P$ and $Q$. The underlying set of $\mathcal{C}$ is the point set $|\mathcal{C}|=\bigcup_{P \in \mathcal{C}} P$. A polyhedral subdivision of a polyhedron $P$ is a polyhedral complex $\mathcal{C}$ with $|\mathcal{C}|=P$.

For a polyhedral convex function $f$, let $\mathcal{T}(f)$ be the polyhedral subdivision of $\operatorname{dom} f$ induced by the projection of lower faces epi $f$, which is represented as

$$
\mathcal{T}(f)=\left\{F \subseteq \mathbf{R}^{n} \mid F \text { is the set of minimizers of } f(\cdot)-\langle p, \cdot\rangle \text { for some } p \in \mathbf{R}^{n}\right\} \text {. }
$$

Such subdivisions are said to be regular; see Chapter 5 of [27]. Note that on each $F \in \mathcal{T}(f), f$ is an affine function.

For two polyhedral subdivisions $\mathcal{C}_{1}$ and $\mathcal{C}_{2}$, the common refinement $\mathcal{C}_{1} \wedge \mathcal{C}_{2}$ is defined by $\mathcal{C}_{1} \wedge \mathcal{C}_{2}=\left\{F \cap G \mid F \in \mathcal{C}_{1}, G \in \mathcal{C}_{2}, F \cap G \neq \emptyset\right\}$. Note that $\mathcal{C}_{1} \wedge \mathcal{C}_{2}$ is a polyhedral subdivision of $\left|\mathcal{C}_{1}\right| \cap\left|\mathcal{C}_{2}\right|$. In particular, for a set of hyperplanes $\mathcal{H}$, we define the polyhedral subdivision $\mathcal{A}(\mathcal{H})$ of $\mathbf{R}^{n}$ as

$$
\mathcal{A}(\mathcal{H})=\bigwedge_{H \in \mathcal{H}}\left\{H, H^{+}, H^{-}\right\} .
$$


Namely, $\mathcal{A}(\mathcal{H})$ is the partition of $\mathbf{R}^{n}$ by hyperplanes $\mathcal{H}$. We give a fundamental relationship between $\mathcal{T}(f+g)$ and $\mathcal{T}(f) \wedge \mathcal{T}(g)$.

Lemma 2.1. Let $f$ and $g$ be polyhedral convex functions with $\operatorname{dom} f \cap \operatorname{dom} g \neq \emptyset$. Then we have

$$
\mathcal{T}(f+g)=\mathcal{T}(f) \wedge \mathcal{T}(g) .
$$

Proof. Both sides of (2.8) are polyhedral subdivisions of $\operatorname{dom} f \cap \operatorname{dom} g$. Hence it is sufficient to show the inclusion (). Let $F \in \mathcal{T}(f)$ and $G \in \mathcal{T}(g)$ be the sets of minimizers of $f(\cdot)-\langle p, \cdot\rangle$ and $g(\cdot)-\langle q, \cdot\rangle$ for some $p, q \in \mathbf{R}^{n}$, respectively. If $F \cap G \neq \emptyset$, then $F \cap G$ is the set of minimizers of $(f+g)(\cdot)-\langle p+q, \cdot\rangle$. Hence $F \cap G \in \mathcal{T}(f+g)$.

\subsection{Polyhedral Split Decomposition}

Here we derive the split decomposition of polyhedral convex functions, which is our guiding principle in deriving the split decomposition of metrics and related concepts in terms of convex analysis and polyhedral geometry. Furthermore, our derivation leads to an algorithm for the discrete version of polyhedral split decomposition in the next section.

First we define a split function, which is a correspondence of a split metric. For a hyperplane $H=H_{a, b}$ with $\|a\|=1$, the split function $l_{H}: \mathbf{R}^{n} \rightarrow \mathbf{R}$ associated with $H$ is defined as

$$
l_{H}(x)=|\langle a, x\rangle-b| / 2 \quad\left(x \in \mathbf{R}^{n}\right) .
$$

For a polyhedral convex function $f$ and a hyperplane $H$, we define the nonnegative number $c_{H}(f) \in \mathbf{R}_{+} \cup\{+\infty\}$ as

$$
c_{H}(f)=\sup \left\{t \in \mathbf{R}_{+} \mid f-t l_{H} \text { is convex }\right\}
$$

and define the set of hyperplane $\mathcal{H}(f)$ as

$$
\mathcal{H}(f)=\left\{H \text { : hyperplane } \mid 0<c_{H}(f)<+\infty\right\} .
$$

Note that $c_{H}(f)=+\infty$ if and only if $\operatorname{dom} f \subseteq H^{+}$or $\operatorname{dom} f \subseteq H^{-}$. The main aim here is to prove the following.

Theorem 2.2. A polyhedral convex function $f: \mathbf{R}^{n} \rightarrow \mathbf{R} \cup\{+\infty\}$ with $\operatorname{dim} \operatorname{dom} f=n$ can be decomposed as

$$
f=\sum_{H \in \mathcal{H}(f)} c_{H}(f) l_{H}+f^{\prime},
$$

where $f^{\prime}$ is a polyhedral convex function with $c_{H}\left(f^{\prime}\right) \in\{0,+\infty\}$ for each hyperplane $H$. Moreover, this representation is unique in the following sense. If $f$ is represented as

$$
f=\sum_{H \in \mathcal{H}} \alpha_{H} l_{H}+g
$$


for some set of hyperplanes $\mathcal{H}$, each of whose elements meets int dom $f$, positive numbers $\left\{\alpha_{H}\right\}_{H \in \mathcal{H}}$, and a polyhedral convex function $g$ with $c_{H}(g) \in\{0,+\infty\}$ for any hyperplane $H$, then we have

$$
\mathcal{H}=\mathcal{H}(f), \quad \alpha_{H}=c_{H}(f) \quad(H \in \mathcal{H}), \quad f^{\prime}=g .
$$

The proof needs three lemmas (Lemmas 2.3-2.5). The first is a convexity condition of a function $f-t l_{H}$. The second is a condition of $0<c_{H}(f)<+\infty$ for $f$ and $H$. The third is a behavior of $c_{H^{\prime}}\left(f-t l_{H}\right)$.

The proof uses the structure of the subdivision $\mathcal{T}(f)$ defined by (2.6). In particular, a full dimensional polyhedron of $\mathcal{T}(f)$ is called a linearity domain of $f$. Note that such members exist since dom $f$ is full dimensional. A function $g: \mathbf{R}^{n} \rightarrow \mathbf{R} \cup\{+\infty\}$ is called a $\mathcal{T}(f)$-piecewise-linear function if $\operatorname{dom} g=\operatorname{dom} f, g$ is continuous on $\operatorname{dom} g$, and is an affine function on each linearity domain of $f$. We shall discuss the convexity condition for $\mathcal{T}(f)$-piecewise-linear function $g$. On each linearity domain $F, g$ can be uniquely represented as

$$
g(x)=\left\langle p_{F}^{g}, x\right\rangle-b_{F}^{g} \quad(x \in F)
$$

for some $\left(p_{F}^{g}, b_{F}^{g}\right) \in \mathbf{R}^{n} \times \mathbf{R}$. A pair of linearity domains $F$ and $G$ is said to be adjacent if $\operatorname{dim} F \cap G=n-1$. In particular, we denote the hyperplane aff $F \cap G$ by $H_{F, G}$ and assume $F \subseteq H_{F, G}^{+}$and $G \subseteq H_{F, G}^{-}$. For two adjacent linearity domains $F, G \in \mathcal{T}(f)$, we define a function $g_{F, G}$ as

$$
g_{F, G}(x)=\left\{\begin{array}{ll}
\left\langle p_{F}^{g}, x\right\rangle-b_{F}^{g} & \text { if } \quad x \in H_{F, G}^{+} \\
\left\langle p_{G}^{g}, x\right\rangle-b_{G}^{g} & \text { if } \quad x \in H_{F, G}^{-}
\end{array} \quad\left(x \in \mathbf{R}^{n}\right) .\right.
$$

In particular, $g$ coincides with $g_{F, G}$ on $F \cup G$. By continuity of $g, g_{F, G}$ can also be represented by

$$
\begin{aligned}
g_{F, G} & = \begin{cases}\max \left\{\left\langle p_{F}^{g}, \cdot\right\rangle-b_{F}^{g},\left\langle p_{G}^{g}, \cdot\right\rangle-b_{G}^{g}\right\} & \text { if } g_{F, G} \text { is convex, } \\
\min \left\{\left\langle p_{F}^{g}, \cdot\right\rangle-b_{F}^{g},\left\langle p_{G}^{g}, \cdot\right\rangle-b_{G}^{g}\right\} & \text { if } g_{F, G} \text { is concave, }\end{cases} \\
& = \begin{cases}\left\|p_{F}^{g}-p_{G}^{g}\right\| l_{H_{F, G}}+\text { affine function } & \text { if } g_{F, G} \text { is convex, } \\
-\left\|p_{F}^{g}-p_{G}^{g}\right\| l_{H_{F, G}}+\text { affine function } & \text { if } g_{F, G} \text { is concave. }\end{cases}
\end{aligned}
$$

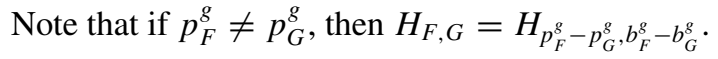

Lemma 2.3. $A \mathcal{T}(f)$-piecewise-linear function $g$ is convex if and only if $g_{F, G}$ is convex for each pair of adjacent linearity domains $F$ and $G$ of $f$.

Proof. The only-if part is obvious by $g=g_{F, G}$ on $F \cup G$. We show the if part. For sufficiently generic points $x, y \in \operatorname{dom} g$, the line segment $[x, y]$ meets only $n$ or $(n-1)$-dimensional members of $\mathcal{T}(f)$. By condition, $g$ is convex on $[x, y]$, i.e., $g$ satisfies (2.4) for $x, y$ and $0 \leq \lambda \leq 1$. Hence, by continuity of $g, g$ satisfies (2.4) for any $x, y \in \operatorname{dom} f$.

Lemma 2.4. For a hyperplane $H=H_{a, b}$ for $\|a\|=1$, we have $0<c_{H}(f)<+\infty$ if and only if $H \cap \operatorname{int} \operatorname{dom} f \neq \emptyset$ and $F \subseteq H^{+}$or $F \subseteq H^{-}$for each $F \in \mathcal{T}(f)$. 
Proof. The only-if part is easily observed. Indeed, if there exists $F \in \mathcal{T}(f)$ such that both $F \cap H^{++}$and $F \cap H^{--}$are nonempty, then $f-t l_{H}$ is not convex on $F$ for any $t>0$. We show the if part. By the condition, $f-t l_{H}$ is a $\mathcal{T}(f)$-piecewise-linear function. By Lemma 2.3, $f-t l_{H}$ is convex if and only if $\left(f-t l_{H}\right)_{F, G}$ is convex for each pair of adjacent linearity domains $F, G$ of $f$. If $F, G \subseteq H^{+}$or $F, G \subseteq H^{-}$, then we have

$$
\left(f-t l_{H}\right)_{F, G}=f_{F, G}+\text { affine function }
$$

and $\left(f-t l_{H}\right)_{F, G}$ is convex for $t \geq 0$. If $F \subseteq H^{+}$and $G \subseteq H^{-}$, then we have aff $F \cap G=H_{F, G}=H_{a, b}$ and $p_{F}^{f}-p_{G}^{f}= \pm\left\|p_{F}^{f}-p_{G}^{f}\right\| a$. Hence we have

$$
\left(f-t l_{H}\right)_{F, G}=\left(\left\|p_{F}^{f}-p_{G}^{f}\right\|-t\right) l_{H}+\text { affine function. }
$$

Hence we have

$$
c_{H}(f)=\min \left\{\left\|p_{F}^{f}-p_{G}^{f}\right\| \mid F, G \text { are linearity domains with aff } F \cap G=H\right\} .
$$

By definition of linearity domain, we have $p_{F}^{f} \neq p_{G}^{f}$ and $\left\|p_{F}^{f}-p_{G}^{f}\right\|>0$. By polyhedrality of $f$, pairs of adjacent linearity domains are finite. Hence we have $0<c_{H}<$ $+\infty$.

Lemma 2.5. For $H, H^{\prime} \in \mathcal{H}(f)$ and $t \in\left[0, c_{H}(f)\right]$, we have

$$
c_{H^{\prime}}\left(f-t l_{H}\right)= \begin{cases}c_{H}(f)-t & \text { if } H^{\prime}=H, \\ c_{H^{\prime}}(f) & \text { otherwise. }\end{cases}
$$

Proof. For distinct $H, H^{\prime} \in \mathcal{H}(f)$, two subsets

$$
\begin{aligned}
& \{\{F, G\}: \text { a pair of linearity domains of } f \text { with aff } F \cap G=H\}, \\
& \left\{\{F, G\}: \text { a pair of linearity domains of } f \text { with aff } F \cap G=H^{\prime}\right\}
\end{aligned}
$$

are disjoint. Hence, (2.22) follows from (2.19), (2.20), and (2.21) in the proof of Lemma 2.4.

The Proof of Theorem 2.2. First note that $\mathcal{H}(f)$ is necessarily finite since $H$ is represented by aff $F \cap G$ for some adjacent linearity domains $F$ and $G$ of $f$ and the set of linearity domains are finite by polyhedrality of $f$. By Lemma $2.5, f^{\prime}=f-\sum_{H \in \mathcal{H}(f)} c_{H}(f) l_{H}$ is convex and $c_{H}\left(f^{\prime}\right) \in\{0,+\infty\}$ for any hyperplane $H$. Hence we obtain the first part. We show the uniqueness part. Suppose that $f$ can be decomposed as the form (2.13). Then we have $\mathcal{H} \subseteq \mathcal{H}(f)$ and $\alpha_{H} \leq c_{H}(f)$ for $H \in \mathcal{H}$. By Lemma 2.5, we have

$$
\begin{aligned}
0 & =c_{H^{\prime}}(g)=c_{H^{\prime}}\left(f-\sum_{H \in \mathcal{H}} \alpha_{H} l_{H}\right) \\
& = \begin{cases}c_{H}(f)-\alpha_{H} & \text { if } H^{\prime}=H \text { for some } H \in \mathcal{H}, \\
c_{H^{\prime}}(f) & \text { otherwise }\end{cases}
\end{aligned}
$$

for $H^{\prime} \in \mathcal{H}(f)$. This implies (2.14). 
Remark 2.6. We observe that $\mathcal{T}\left(\alpha l_{H}\right)=\left\{H, H^{+}, H^{-}\right\}$for $\alpha \in \mathbf{R}_{++}$. Hence, by Lemma 2.1, corresponding to the decomposition (2.12), $\mathcal{T}(f)$ is decomposed as

$$
\mathcal{T}(f)=\mathcal{A}(\mathcal{H}(f)) \wedge \mathcal{T}\left(f^{\prime}\right) .
$$

Then, by Lemma 2.4, there is no hyperplane $H$ meeting int dom $f$ such that $F \subseteq H^{+}$ and $F \subseteq H^{-}$for each $F \in \mathcal{T}\left(f^{\prime}\right)$. This gives a geometric interpretation of the splitprimeness of metrics in Section 4.

Closing this subsection, we give an alternative expression of $c_{H}(f)$, which will be used in Section 3.

Lemma 2.7. For a polyhedral convex function $f: \mathbf{R}^{n} \rightarrow \mathbf{R} \cup\{+\infty\}$ with $\operatorname{dim} \operatorname{dom} f=$ $n$ and a hyperplane $H$, we have

$$
c_{H}(f)=\frac{1}{2} \inf \left\{\begin{array}{l|l}
\frac{f(x)-f(w)}{l_{H}(x)}+\frac{f(y)-f(w)}{l_{H}(y)} & \begin{array}{l}
x \in \operatorname{dom} f \cap H^{++} \\
y \in \operatorname{dom} f \cap H^{--} \\
\{w\}=[x, y] \cap H
\end{array}
\end{array}\right\} .
$$

In particular, if $c_{H}(f)=0$, then there exists some linearity domain $F$ of $f$ such that both $F \cap H^{++}$and $F \cap H^{--}$are nonempty and any $x \in F \cap H^{++}$and $y \in F \cap H^{--}$attain the infimum of the right-hand side of (2.27). If $0<c_{H}(f)<+\infty$, then there exists an adjacent pair of linearity domain $F \subseteq H^{+}, G \subseteq H^{-}$of $f$ such that $\operatorname{aff}(F \cap G)=H$ and any $x \in F \cap H^{++}$and $y \in G \cap H^{--}$attain the infimum of the right-hand side of (2.27).

Proof. First we show $c_{H}(f) \leq$ RHS of (2.27). Indeed, since $f-c_{H}(f) l_{H}$ is convex, $f-c_{H}(f) l_{H}$ satisfies (2.4) for $x \in H^{++}, y \in H^{--}$, and $\lambda=l_{H}(y) /\left(l_{H}(x)+l_{H}(y)\right)$ as

$$
\lambda\left(f-c_{H}(f) l_{H}\right)(x)+(1-\lambda)\left(f-c_{H}(f) l_{H}\right)(y) \leq\left(f-c_{H}(f)\right)(\lambda x+(1-\lambda) y) .
$$

Put $w=\lambda x+(1-\lambda) y$. Then we have $\{w\}=H \cap[x, y]$ and $l_{H}(w)=0$. Hence we obtain

$$
c_{H}(f) \leq \frac{1}{2}\left\{\frac{f(x)-f(w)}{l_{H}(x)}+\frac{f(y)-f(w)}{l_{H}(y)}\right\} .
$$

Therefore, it suffices to show the latter part. The statement for the case $c_{H}(f)=0$ follows from Lemma 2.4. In the case $0<c_{H}(f)<+\infty$, by (2.18) and (2.21), there exists a pair of adjacent linearity domains $F \subseteq H^{+}, G \subseteq H^{-}$of $f$ such that

$$
f_{F, G}=c_{H}(f) l_{H}+\text { affine function. }
$$

Furthermore, $F \cup G$ is a linearity domain of $f-c_{H}(f) l_{H}$ and therefore is convex. Then, for any $x \in F \cap H^{++}, y \in G \cap H^{--}$, we have $\{w\}=[x, y] \cap H \in F \cup G$ and

$$
\begin{aligned}
\operatorname{RHS} \text { of (2.29) } & =\frac{1}{2}\left\{\frac{f_{F, G}(x)-f_{F, G}(w)}{l_{H}(x)}+\frac{f_{F, G}(y)-f_{F, G}(w)}{l_{H}(y)}\right\} \\
& =c_{H}(f) .
\end{aligned}
$$




\subsection{Dual View of Theorem 2.2}

In this subsection we discuss the dual view of Theorem 2.2. In particular, we discuss the relationship between Theorem 2.2 and the maximum zonotopic summand of a polyhedron. For this, we focus on the case that $f$ is positively homogeneous, or, equivalently, is the support function of some polyhedron. Recall the relationship between positively homogeneous convex functions and the support functions of a polyhedron; see Section 1.7 of [22] and Section 19 of [21].

For a polyhedron $P$, the support function $f_{P}$ is defined as

$$
f_{P}(x)=\sup \{\langle x, p\rangle \mid p \in P\} \quad\left(x \in \mathbf{R}^{n}\right) .
$$

Then $f_{P}$ is polyhedral convex and positively homogeneous, i.e., $f$ satisfies

$$
f(\lambda x)=\lambda f(x), \quad \lambda \in \mathbf{R}_{+}, \quad x \in \mathbf{R}^{n} .
$$

Conversely, if $f$ is positively homogeneous polyhedral convex, then $f$ is the support function of the polyhedron

$$
P_{f}=\left\{p \in \mathbf{R}^{n} \mid\langle p, x\rangle \leq f(x)\left(x \in \mathbf{R}^{n}\right)\right\} .
$$

This correspondence is one to one and satisfies

$$
P_{f+g}=P_{f}+P_{g}, \quad f_{P+Q}=f_{P}+f_{Q}
$$

for two positively homogeneous polyhedral convex functions $f$ and $g$ and two polyhedra $P$ and $Q$. Note that the split function $l_{H}$ for a linear hyperplane $H=H_{a, 0}$ with $\|a\|=1$ is the support function of the line segment $\frac{1}{2}[-a, a]$.

In this case, $\mathcal{T}\left(f_{P}\right)$ coincides with the normal fan of $P$, which consists of the normal cones of $P_{f}$. Recall the definition of the normal cone; see Chapter 7 of [27] and Section 2.4 of [22]. For a face $F$ of $P_{f}$, the normal cone $N_{F}$ of $F$ is defined as

$$
N_{F}=\left\{x \in \mathbf{R}^{n} \mid\{q \in P \mid\langle x, q\rangle=\sup \{\langle x, p\rangle \mid p \in P\}\} \subseteq F\right\}
$$

and hence we have $\mathcal{T}\left(f_{P}\right)=\left\{N_{F} \mid F\right.$ is a face of $\left.P\right\}$. Note that $\operatorname{dom} f_{P}$ is given by $\left\{x \in \mathbf{R}^{n} \mid\langle p, x\rangle \leq 0(p \in \operatorname{rec} P)\right\}$, where rec $P$ is the recession cone of $P$ defined by $\left\{v \in \mathbf{R}^{n} \mid P+[0, v]=P\right\}$.

In particular, faces of $P_{f}$ and $\mathcal{T}(f)$ are in polar relation as follows:

(N1) $F \subseteq G$ if and only if $N_{F} \supseteq N_{G}$, and

(N2) the linear subspace parallel to aff $F$ is orthogonal to aff $N_{F}$,

where $F$ and $G$ are faces of $P_{f}$. In addition, we see the following relation:

(N3) $F$ is bounded if and only if $N_{F} \cap$ int $\operatorname{dom} f \neq \emptyset$.

Applying Theorem 2.2 to the support function of a polyhedron $P$, we have the following, which can be understood as an unbounded refinement of theorem of Bolker [4, p. 341] that every polytope (bounded polyhedron) has the maximum zonotopic summand, where a polyhedron $Q$ is called a Minkowski summand of a polyhedron $P$ if there exists a polyhedron $P^{\prime}$ such that $P=Q+P^{\prime}$, and $P$ is said to be pointed if $P$ has a vertex. 
Corollary 2.8. A pointed polyhedron $P$ is uniquely decomposed as

$$
P=\sum_{v \in \mathcal{V}}[-v / 2, v / 2]+P^{\prime}
$$

for a finite set of vectors $\mathcal{V} \subseteq \mathbf{R}^{n} \backslash \pm \operatorname{rec} P$ and a polyhedron $P^{\prime}$ which does not contain $[-u / 2, u / 2]$ as a Minkowski summand for each $u \in \mathbf{R}^{n} \backslash \pm \operatorname{rec} P$.

Proof. Let $f_{P}$ be the support function of $P$. Note that dom $f_{P}$ is full dimensional since $P$ is pointed. Since $\mathcal{T}(f)$ consists of polyhedral cones, $\mathcal{H}(f)$ consists of linear hyperplanes. From $\operatorname{dom} f_{P}=\left\{x \in \mathbf{R}^{n} \mid\langle p, x\rangle \leq 0(p \in \operatorname{rec} P)\right\}$, we have $\operatorname{dom} f_{P} \subseteq H_{a, 0}^{+}$or $\operatorname{dom} f_{P} \subseteq H_{a, 0}^{-}$if and only if $a \in \operatorname{rec} P$ or $-a \in \operatorname{rec} P$. Applying Theorem 2.2 to $f_{P}$ and (2.35), we obtain the desired result.

A finite (Minkowski) sum of line segments is called a zonotope. The zonotope $Z(P)=$ $\sum_{v \in \mathcal{V}}[-v / 2, v / 2]$ of $(2.37)$ is called the maximum zonotopic summand of $P$. As will be seen in Section 4, the split decomposition of metrics can be understood as the extraction of the maximum zonotopic summand from some polyhedron associated with a given metric; see Remark 4.10.

The structure of the union of the bounded faces of $P_{f}$ expresses the nonlinearity of $f$ over $\operatorname{dom} f$. For example, $f$ is linear on $\operatorname{dom} f$ if and only if the union of the bounded faces of $P_{f}$ is a single point. As will be shown in Section 4, the following proposition gives an interpretation and another proof to the result of Dress [7] that a metric is a tree metric if and only if its tight span is a tree.

Proposition 2.9. For a positively homogeneous polyhedral convex function $f: \mathbf{R}^{n} \rightarrow$ $\mathbf{R} \cup\{+\infty\}$ with $\operatorname{dim} \operatorname{dom} f=n$, the following conditions are equivalent:

(1) The dimension of the union of bounded faces of $P_{f}$ is (less than or) equal to 1.

(2) The union of bounded faces of $P_{f}$ is a tree.

(3) If $f$ is decomposed as (2.12), then $f^{\prime}$ is linear over $\operatorname{dom} f$ and $H_{1} \cap H_{2} \cap$ int dom $f=\emptyset$ for each pair $H_{1}, H_{2} \in \mathcal{H}(f)$.

Proof. The equivalence (1) $\Leftrightarrow(2)$ follows from the general fact that the union of bounded faces of a polyhedron is contractible, i.e., it is homotopy equivalent to a point (see the Appendix of [15] for a proof). We show (3) $\Rightarrow(1)$. By condition, each member of $\mathcal{T}(f)$ meeting int dom $f$ has the dimension $n$ or $n-1$. By (N2), we have (1). Finally we show (2) $\Rightarrow(3)$. Let $E$ be a one-dimensional bounded face of $P_{f}$. Then $F$ is a line segment. By (N2), $N_{E}$ is an $(n-1)$-dimensional member of $\mathcal{T}(f)$. We claim that aff $N_{E} \cap \operatorname{dom} f_{P}=N_{E}$. Indeed, this follows from the fact that every proper face of $N_{E}$ is contained in the boundary of $\operatorname{dom} f$ by (N2). By Lemma 2.4, we conclude that (linear) hyperplanes $\left\{\right.$ aff $N_{E} \mid E$ is a bounded edge of $\left.P\right\}=\mathcal{H}(f)$ and do not intersect in int dom $f$ each other. Moreover, $N_{E}$ can be uniquely represented by $F \cap G$ for two adjacent linearity domains $F$ and $G$. Then $F \cup G$ is a linearity domain of $f-c_{H} l_{H}$. Repeat this process to $f-c_{H}(f) l_{H}$, then $\operatorname{dom} f$ is a linearity domain of $f-\sum_{H \in \mathcal{H}(f)} c_{H}(f) l_{H}$. This implies that $f-\sum_{H \in \mathcal{H}(f)} c_{H}(f) l_{H}$ is linear over $\operatorname{dom} f$. 


\section{Split Decomposition of Discrete Functions}

In this section we describe a discrete version of the polyhedral split decomposition, which is a certain kind of decomposition of a function $f$ defined on a finite set $X$ of points of $\mathbf{R}^{n}$. The basic idea is to associate $f$ to some polyhedral convex function $\bar{f}$ as in Fig. 1 in the Introduction and apply Theorem 2.2.

\subsection{Discrete Functions and Convex-Extension}

Let $X$ be a finite point set of $\mathbf{R}^{n}$. We assume that $X$ is contained by some affine hyperplane $K$ not containing the origin of $\mathbf{R}^{n}$. If necessary, we consider $\tilde{X}=\{(y, 1) \mid y \in X\} \subseteq$ $\mathbf{R}^{n+1}$ instead of $X$; in this case $K=\left\{(x, 1) \mid x \in \mathbf{R}^{n}\right\} \subseteq \mathbf{R}^{n+1}$. For a function $f: X \rightarrow \mathbf{R}$, we consider the following polyhedral convex function:

$$
\bar{f}(x)=\inf \left\{\sum_{y \in X} \lambda_{y} f(y) \mid \sum_{y \in X} \lambda_{y} y=x, \lambda_{y} \geq 0(y \in X)\right\},
$$

which is called the homogeneous convex extension of $f$. Then, by linear programming duality, $\bar{f}$ is the support function of the polyhedron

$$
Q(f)=\left\{p \in \mathbf{R}^{n} \mid\langle p, y\rangle \leq f(y)(y \in X)\right\}
$$

and, therefore, positively homogeneous. $\operatorname{dom} \bar{f}$ is given by cone $X$. Then $\mathcal{T}(\bar{f})$ is the normal fan of $Q(f)$. Let $\mathcal{T}^{X}(\bar{f})$ denote the subdivision of conv $X$ which is defined by $\{F \cap K \mid F \in \mathcal{T}(\bar{f})\}$. Note that the restriction of $\bar{f}$ to $K$ is the function of the lower envelope of the convex hull of $\{(y, f(y)) \mid y \in X\} \subseteq K \times \mathbf{R}$ and $\mathcal{T}^{X}(\bar{f})$ is the projection of lower faces of $\operatorname{conv}\{(y, f(y)) \mid y \in X\}$; recall Fig. 1 in the Introduction. For a function $g: \mathbf{R}^{n} \rightarrow \mathbf{R} \cup\{+\infty\}$, we denote the restriction of $g$ to $X$ by $g^{X} . f$ is said to be convex-extensible if it satisfies $\bar{f}^{X}=f$. The set of convex-extensible functions is recognized as a fundamental class of the discrete convex functions (see [20]).

We give some fundamental properties of discrete functions and homogeneous convexextensions, which will be used in the proof of Theorem 3.4 and Proposition 3.6 in the next subsection.

Lemma 3.1. Let $f, g: X \rightarrow \mathbf{R}$ be a function.

(1) $\overline{c f+\langle q, \cdot\rangle}=c \bar{f}+\langle q, \cdot\rangle+\delta_{\text {cone } X}\left(c \in \mathbf{R}_{+}, q \in \mathbf{R}^{n}\right)$, where $\delta_{\text {cone } X}$ is the indicator function of cone $X$; see (2.5).

(2) $F \in \mathcal{T}(\bar{f})$ is represented as cone $\{y \mid y \in X,\langle q, y\rangle=f(y)\}$ for some $q \in Q(f)$. Furthermore $\bar{f}(x)=\overline{f^{F \cap X}}(x)$ for $x \in F$. In particular, $\bar{f}(y)=f(y)$ if $y \in X$ is a vertex of $\mathcal{T}^{X}(\bar{f})$.

Proof. (1) By (3.2), we have

$$
\overline{c f+\langle q, \cdot\rangle}(x)=\sup \left\{\langle x, p\rangle \mid p \in \mathbf{R}^{n},\langle p, y\rangle \leq c f(y)+\langle q, y\rangle(y \in X)\right\} .
$$


If $c>0$, substitute $p^{\prime}=(p-q) / c$ to the right-hand side of (3.3). Then we have the desired result. If $c=0$, then it is immediate from definition (3.1).

(2) The first statement follows from the fact that a normal cone of $Q(f)$ can be represented as the conical combination of the normal vectors of facets of $Q(f)$ containing some $q \in Q(f)$. Next we show $\bar{f}(x)=\bar{f}^{F \cap X}(x)$ for $x \in F$. Indeed, the coefficient $\lambda$ of conical combination $x=\sum_{y \in X,\langle q, y\rangle=f(y)} \lambda_{y} y$ and $q$ satisfies the feasibility and the complementary slackness condition for the dual pairs of linear programs corresponding to $\bar{f}(x)$ and $\overline{f^{F \cap X}}(x)$. Hence we obtain $\bar{f}(x)=\bar{f}^{F \cap X}(x)=\sum_{y \in X,\langle q, y\rangle=f(y)} \lambda_{y} f(y)$; see [23] for linear programming.

\subsection{Split Decomposition of Discrete Functions}

Here, we present the discrete version of Theorem 2.2. As shown in the next section, this turns out to coincide with (an extension of) Bandelt-Dress' split decomposition in the case of $X=\Lambda$. Furthermore, some interesting properties of weakly compatible splits are also generalized. Arguments in this subsection have their own significance in the theory of regular subdivision; see Remark 3.8. Note that since $\mathcal{T}(\bar{f})$ for $f: X \rightarrow \mathbf{R}$ is the normal fan of $Q(f)$, each hyperplane $H \in \mathcal{T}(\bar{f})$ is linear, i.e., $H=H_{a, 0}$ for some $a \in \mathbf{R}^{n}$. We assume that aff $X=K$, which guarantees the full dimensionality of $\operatorname{dom} \bar{f}=$ cone $X$ for $f \in \mathbf{R}^{X}$. The discrete version of Theorem 2.2 is as follows.

Theorem 3.2. A discrete function $f: X \rightarrow \mathbf{R}$ can be decomposed as

$$
f=\sum_{H \in \mathcal{H}(\bar{f})} c_{H}(\bar{f}) l_{H}^{X}+\gamma,
$$

where $\gamma: X \rightarrow \mathbf{R}$ satisfies $c_{H^{\prime}}(\bar{\gamma}) \in\{0,+\infty\}$ for any linear hyperplane $H^{\prime}$. Moreover, we have

$$
\bar{f}=\sum_{H \in \mathcal{H}(\bar{f})} c_{H}(\bar{f}) l_{H}+\bar{\gamma}
$$

or, equivalently,

$$
Q(f)=\sum_{H \in \mathcal{H}(\bar{f})} c_{H}(\bar{f})\left[-n_{H} / 2, n_{H} / 2\right]+Q(\gamma),
$$

where $n_{H}$ is the unit normal vector of hyperplane $H$. If, in addition, $f$ is convexextensible, then $\gamma$ is also convex-extensible.

Proof. We can apply Theorem 2.2 to $\bar{f}$ by $\operatorname{dim} \bar{f}=\operatorname{dim}$ cone $X=n$. It suffices to show $\bar{f}=t l_{H}+\overline{\left(f-t l_{H}^{X}\right)}$, or, equivalently,

$$
Q(f)=t\left[n_{H} / 2,-n_{H} / 2\right]+Q\left(f-t l_{H}^{X}\right)
$$

for $H \in \mathcal{H}(\bar{f})$, and $t \in\left[0, c_{H}(\bar{f})\right]$. We show (3.7). The inclusion () follows from

$$
\left\langle p+s n_{H} / 2, y\right\rangle \leq f(y)-t\left|\left\langle n_{H}, y\right\rangle\right| / 2+s\left\langle n_{H}, y\right\rangle / 2 \leq f(y) \quad(y \in X)
$$




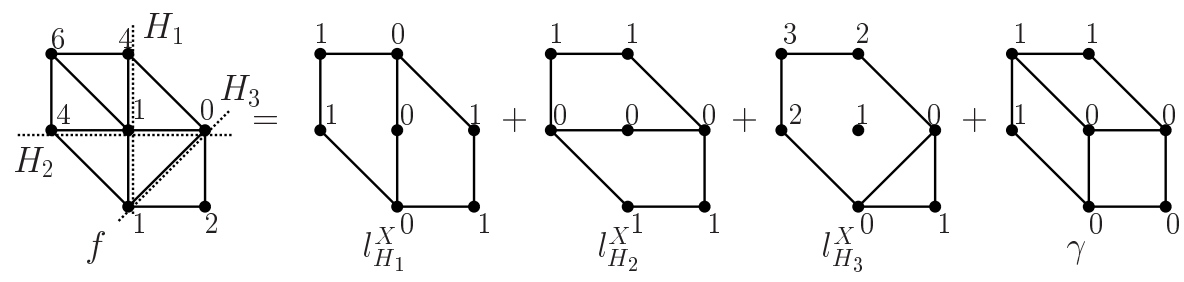

Fig. 4. The split decomposition of $f$.

for $s \in[-t, t]$ and $p \in Q\left(f-t l_{H}^{X}\right)$. We next show $(\subseteq)$. By $\bar{f}=t l_{H}+\left(\bar{f}-t l_{H}\right)$ and (2.35), we have

$$
Q(f)=P_{\bar{f}}=t\left[n_{H} / 2,-n_{H} / 2\right]+P_{\bar{f}-t l_{H}} .
$$

By the definition of $Q(\cdot)(3.2),(2.34)$, and $\bar{f}^{X} \leq f$, we have

$$
P_{\bar{f}-t l_{H}} \subseteq Q\left(\left(\bar{f}-t l_{H}\right)^{X}\right) \subseteq Q\left(f-t l_{H}^{X}\right) .
$$

Hence, we obtain $(\subseteq)$. If $f$ is convex-extensible, then $\bar{f}^{X}=f$. By restricting both sides of (3.10) to $X$, we have $\gamma=\bar{\gamma}^{X}$. Hence $\gamma$ is convex-extensible.

Figure 4 is an example of the split decomposition (3.4) of a function $f: X \rightarrow \mathbf{R}$ for $X \subseteq \mathbf{R}^{3}$ in terms of subdivisions induced by each term in (3.4), where this figure illustrates the restriction to two-dimensional space $K=\operatorname{aff} X$, each black point denotes a point of $X$, and the number near each black point denotes corresponding value of $f$. The property (3.5) (or (3.6)) means that (3.4) decomposes $f$ to the sum of functions each of which induces the subdivision coarser than $\mathcal{T}^{X}(\bar{f})$. This property corresponds to the coherence property of additive decomposition of metrics; see Section 4.

From Fig. 4 we observe that possible hyperplanes appearing in $\mathcal{H}(\cdot)$ are limited by the point set $X$. Motivated by this observation, we define a certain geometric condition of hyperplane arrangements, which is determined solely by $X$, as follows. A set of linear hyperplane $\mathcal{H}$ is $X$-admissible if $\mathcal{H}$ satisfies

(A0) $H \cap$ ri conv $X \neq \emptyset$ for each $H \in \mathcal{H}$, and

(A1) $\operatorname{conv}(F \cap X)=F \cap \operatorname{conv} X$ for each $F \in \mathcal{A}(\mathcal{H})$.

In particular, condition (A1) can be rephrased as

$$
\{\text { vertices of }(\mathcal{A}(\mathcal{H}) \wedge\{\text { faces of conv } X\})\} \subseteq X .
$$

Namely, when $\mathcal{H}$ cuts conv $X$, newly appeared vertices are contained in $X$.

Lemma 3.3. For $f: X \rightarrow \mathbf{R}$, the set of hyperplanes $\mathcal{H}(\bar{f})$ is $X$-admissible.

Proof. Condition (A0) is clearly satisfied. We show (A1). The inclusion (ㄷ) is obvious. We show (). By (2.26) and Lemma 3.1(2), we have

$$
F \cap \operatorname{conv} X=\bigcup\left\{G \in \mathcal{T}^{X}(\bar{f}) \mid G \subseteq F\right\}
$$




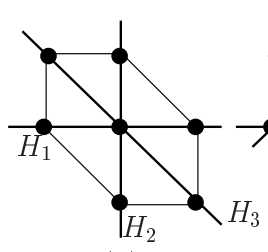

(a)

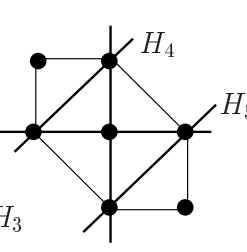

(b)

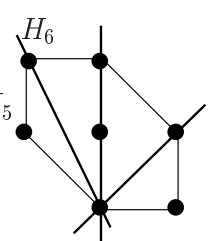

(c)

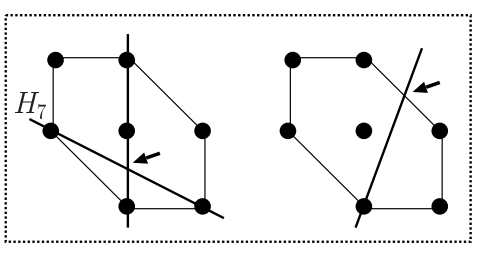

(d)

(e)

Fig. 5. $X$-admissibility of hyperplanes.

$$
\begin{aligned}
& =\bigcup\left\{\operatorname{conv}(G \cap X) \mid G \in \mathcal{T}^{X}(\bar{f}), G \subseteq F\right\} \\
& \subseteq \operatorname{conv}\left(X \cap \bigcup\left\{G \in \mathcal{T}^{X}(\bar{f}) \mid G \subseteq F\right\}\right) \\
& =\operatorname{conv}(X \cap F) . \square
\end{aligned}
$$

Figure 5 illustrates examples of $X$-admissible and non- $X$-admissible sets of hyperplanes, where black points are $X$, and hyperplanes of (a)-(c) are $X$-admissible, and (d) and (e) are not since there are vertices, indicated by the arrow, not contained by $X$. Note that if $\mathcal{H}$ is $X$-admissible, then any subset of $\mathcal{H}$ is also $X$-admissible. So we define the set of linear hyperplanes $\mathcal{H}_{X}$ as

$$
\mathcal{H}_{X}=\{H \text { : linear hyperplane } \mid\{H\} \text { is } X \text {-admissible }\} \text {. }
$$

By (A0), (A1), and (3.11), we see that $H \in \mathcal{H}_{X}$ if and only if $H$ satisfies

(a0) there exists $x, y \in X$ such that $x \in H^{++}$and $y \in H^{--}$, and

(a1) for each edge $[x, y]$ of conv $X$ with $x \in H^{++}, y \in H^{--}$, we have $[x, y] \cap H \in X$.

Note that $\mathcal{H}_{X}$ is a finite set since $H \in \mathcal{H}_{X}$ is represented as the linear space spanned by some $Y \subseteq X$. An $X$-admissible set of hyperplanes is a subset of $\mathcal{H}_{X}$. The next theorem implies that the discrete split decomposition (3.4) can be carried out without explicit construction of convex-extensions; $c_{H}(\bar{f})$ can be calculated discretely.

Theorem 3.4. For a function $f: X \rightarrow \mathbf{R}$ and a hyperplane $H \in \mathcal{H}_{X}$, let $\tilde{c}_{H}(f)$ be defined by

$$
\frac{1}{2} \min \left\{\frac{f(x)-\overline{f^{X \cap H}}(w)}{l_{H}(x)}+\frac{f(y)-\overline{f^{X \cap H}}(w)}{l_{H}(y)} \mid \begin{array}{c}
x \in X \cap H^{++} \\
y \in X \cap H^{--} \\
\{w\}=H \cap[x, y]
\end{array}\right\} .
$$

Then we have

$$
c_{H}(\bar{f})=\max \left(0, \tilde{c}_{H}(f)\right) .
$$

Proof. By Lemmas 2.7 and 3.1(2), $c_{H}(\bar{f})$ can be represented as

$$
c_{H}(\bar{f})=\frac{1}{2} \min \left\{\frac{f(y)-\bar{f}(w)}{l_{H}(y)}+\frac{f(z)-\bar{f}(w)}{l_{H}(z)} \mid \begin{array}{c}
y \in X \cap H^{++} \\
z \in X \cap H^{--} \\
\{w\}=H \cap[x, y]
\end{array}\right\} .
$$


Since $\overline{f^{X \cap H}}(w) \geq \bar{f}(w)$ holds for $w \in H \cap$ cone $X$, we have $c_{H}(\bar{f}) \geq \tilde{c}_{H}(f)$. If $c_{H}(\bar{f})>0$ holds, then, for $w \in H \cap$ cone $X$, there exists $F \in \mathcal{T}(\bar{f})$ such that $w \in F$ and $F \subseteq H$ by Lemma 2.4. Therefore, by Lemma 3.1(2), we have $\bar{f}(w)=\overline{f^{X \cap F}}(w)=$ $\overline{f^{X \cap H}}(w)$ and $c_{H}(\bar{f})=\tilde{c}_{H}(f)$.

The theorem yields an algorithm for the split decomposition of $f: X \rightarrow \mathbf{R}$ as follows:

1. Determine $\mathcal{H}_{X}$ from the points $X$.

2. Calculate $c_{H}(\bar{f})$ for $H \in \mathcal{H}_{X}$ by formulas (3.14) and (3.15).

3. Decompose $f$ into the form of (3.4).

In Section 4 we derive Bandelt-Dress' split decomposition from this recipe. As will be shown in Section 4, Theorem 4.14, the $X$-admissibility of hyperplanes corresponds to the weak compatibility of splits. The next proposition corresponds to Corollary 10 of [1]. This also gives a criterion for the $X$-admissibility of hyperplanes.

Proposition 3.5. For $\mathcal{H} \subseteq \mathcal{H}_{X}$ and $\alpha \in \mathbf{R}_{++}^{\mathcal{H}}$, let $f=\sum_{H \in \mathcal{H}} \alpha_{H} l_{H}^{X}$. Then the following conditions are equivalent:

(a) $\bar{f}=\sum_{H \in \mathcal{H}} \alpha_{H} l_{H}+\delta_{\text {cone } X}$.

(b) $\mathcal{H}=\mathcal{H}(\bar{f})$ and $\alpha_{H}=c_{H}(\bar{f})$ for $H \in \mathcal{H}$.

(c) $\mathcal{H}$ is $X$-admissible.

Proof. (a) $\Leftrightarrow$ (b) follows from Theorems 2.2 and 3.2. (b) $\Rightarrow$ (c) follows from Lemma 3.3.

(c) $\Rightarrow$ (a) Let $\mathcal{H}=\left\{H_{a_{i}, 0} \mid i \in I\right\}$ with $\left\|a_{i}\right\|=1(i \in I)$. For $x \in$ cone $X$, there exists $F \in \mathcal{A}(\mathcal{H})$ such that $x \in F$. By condition (c), $x$ can be represented as a conical combination

$$
x=\sum_{y \in F \cap X} \lambda_{y} y .
$$

Then the coefficient $\lambda$ is feasible to the corresponding linear program (3.1). On the other hand, $p$ defined as

$$
p=\sum_{i \in I, F \subseteq H_{i}^{+}}\left(\alpha_{H_{i}} / 2\right) a_{i}-\sum_{i \in I, F \subseteq H_{i}^{-}}\left(\alpha_{H_{i}} / 2\right) a_{i}
$$

is also feasible to the dual linear program: $\max$. $\langle p, x\rangle$ s.t. $p \in Q(f)$. It is easy to check that $\lambda$ and $p$ satisfy the complementary slackness condition. This implies (a).

In particular, from the equivalence between (b) and (c) of this proposition, we see that the decomposition into a sum of $X$-admissible split functions is uniquely determined. The number of $X$-admissible hyperplanes is bounded by $|X|-n$. This fact corresponds to Corollary 4 of [1].

Proposition 3.6. Let $\mathcal{H} \subseteq \mathcal{H}_{X}$ be an $X$-admissible set of hyperplanes. Then the set of vectors $\left\{l_{H}^{X} \mid H \in \mathcal{H}\right\} \cup\left\{e_{i}^{X} \mid 1 \leq i \leq n\right\}$ is linearly independent in $\mathbf{R}^{X}$, where $e_{i}: \mathbf{R}^{n} \rightarrow \mathbf{R}$ is the $i$ th coordinate function defined by $e_{i}(x)=x_{i}$ for $x \in \mathbf{R}^{n}$. Therefore $|\mathcal{H}| \leq|X|-n$. 
Proof. Suppose that it is linearly dependent. Then there exists nonzero $(\alpha, p) \in \mathbf{R}^{\mathcal{H}} \times$ $\mathbf{R}^{n}$ such that

$$
\sum_{H \in \mathcal{H}, \alpha_{H}>0} \alpha_{H} l_{H}^{X}+\langle p, \cdot\rangle=\sum_{H \in \mathcal{H}, \alpha_{H}<0}-\alpha_{H} l_{H}^{X} .
$$

By Proposition 3.5 and Lemma 3.1(1), the convex extensions of both sides of (3.19) lead to a contradiction to the uniqueness of the polyhedral split decomposition (Theorem 2.2).

A function $f \in \mathbf{R}^{X}$ is said to be split-decomposable if $f-\sum_{H \in \mathcal{H}(\bar{f})} c_{H}(\bar{f}) l_{H}^{X}$ is (the restriction of) a linear function. By Proposition 3.6, the set of all split-decomposable functions on $X$ can be naturally regarded as a simplicial fan of $\mathbf{R}^{X}$, which is isomorphic to the set of $X$-admissible sets of hyperplanes (as an abstract simplicial complex). If an $X$-admissible $\mathcal{H}$ has the maximal cardinality $|X|-n$, the cone

$$
\left\{\sum_{H \in \mathcal{H}} \alpha_{H} l_{H}^{X}+g^{X} \mid \alpha \in \mathbf{R}_{+}^{\mathcal{H}}, g \text { : linear function on } \mathbf{R}^{n}\right\},
$$

as a subset of $\mathbf{R}^{X}$, has interior points. Therefore, for sufficiently generic $f: X \rightarrow \mathbf{R}$ from the cone (3.20), $\mathcal{T}(\bar{f})$ forms a simplicial fan (see Chapter 7 of [14]).

Corollary 3.7. Let $\mathcal{H} \subseteq \mathcal{H}_{X}$ be an $X$-admissible set of hyperplanes with maximal cardinality $|X|-n$ and let $f=\sum_{H \in \mathcal{H}} l_{H}^{X}$. Then $\mathcal{T}^{X}(\bar{f})$ is a triangulation. Furthermore, the set of vertices of $\mathcal{T}^{X}(\bar{f})$ coincides with $X$.

Proof. The latter part is immediate from the fact that if $y \in X$ is not a vertex of $\mathcal{T}^{X}(\bar{f})$, then $\mathcal{H}$ is also $(X \backslash\{y\})$-admissible.

Figure 5(b) attains the maximum number of $X$-admissible hyperplanes and therefore is a triangulation having vertices $X$.

Remark 3.8. We discuss the significance of the arguments in this section from the viewpoint of the theory of regular subdivisions and secondary fans; see [3] and Chapter 7 of [14] for details. Consider the equivalence relation on $\mathbf{R}^{X}$ so that two functions $f, g \in \mathbf{R}^{X}$ are defined to be equivalent if two subdivisions of $X$ induced by $f$ and $g$ coincide. Then $\mathbf{R}^{X}$ is partitioned into the equivalence classes and each equivalence class is a relatively open polyhedral cone. The closure of this equivalence class is called a secondary cone. Let $\Sigma(X)$ be the set of secondary cones. Then $\Sigma(X)$ is a polyhedral fan and is called the secondary fan of $X$. Let $\Pi(X)$ be the fan of split-decomposable functions on $X$. By Propositions 3.5 and 3.6, each cone of $\Pi(X)$ is a simplicial subset of some secondary cone. Therefore, $\Pi(X)$ is a simplicial subfan of $\Sigma(X)$. See also p. 15 of [26] for a related discussion.

Remark 3.9. We discuss the connection between the arguments in this section and Erdahl and Ryshkov's theory of lattice dicing [10]. For a relatively prime integer vector 
$z \in \mathbf{Z}^{n}$, we define the (infinite) set of parallel hyperplanes $D_{z}$ by $\left\{H_{z, b} \mid b \in \mathbf{Z}\right\}$, where $\mathbf{Z}$ denotes the set of integer vectors. A finite set of parallel hyperplanes ( $D$-family) $\left\{D_{z_{1}}, D_{z_{2}}, \ldots, D_{z_{r}}\right\}$ is called a lattice dicing with respect to $\mathbf{Z}^{n}$ if the set of vertices of the subdivision $\mathcal{A}\left(\bigcup_{i=1}^{r} D_{z_{i}}\right)$ coincides with $\mathbf{Z}^{n}$, i.e., $\bigcup_{i=1}^{r} D_{z_{i}}$ is $\mathbf{Z}^{n}$-admissible in our sense. A positive definite quadratic form $x^{\top} A x$ yields a (infinite) polyhedral subdivision to $\mathbf{R}^{n}$ by projecting the lower faces of the convex hull of $\left\{\left(x, x^{\top} A x\right) \mid x \in \mathbf{Z}^{n}\right\} \subseteq \mathbf{R}^{n} \times \mathbf{R}$, which is called an L-partition. Analogously to the case of secondary fans (Remark 3.8), we define the equivalence relation on the cone of positive definite quadratic forms so that two positive definite forms are defined to be equivalent if the corresponding $L$ partitions coincide. Then the cone of positive definite quadratic forms is partitioned into equivalence classes called $L$-type domains, which are known to be relatively open polyhedral cones. The domain of lattice dicing $\left\{D_{z_{1}}, D_{z_{2}}, \ldots, D_{z_{r}}\right\}$ is a set of positively definite forms represented by $\sum_{i=1}^{r} \lambda_{i}\left(\left\langle d_{i}, x\right\rangle\right)^{2}$ for positive weights $\left\{\lambda_{i}\right\}_{i=1}^{r}$. Erdahl and Ryshkov [10] show that $L$-partition induced by $\sum_{i=1}^{r} \lambda_{i}\left(\left\langle d_{i}, x\right\rangle\right)^{2}$ coincides with $\mathcal{A}\left(\bigcup_{i=1}^{r} D_{z_{i}}\right)$, and each dicing domain is a simplicial $L$-type domain. So the fan of (the closure of) dicing domains can be understood as a correspondence of the fan of split-decomposable functions. Indeed, quadratic form $\left(\left\langle d_{i}, x\right\rangle\right)^{2}$ on $\left\{x \in \mathbf{Z}^{n} \mid-m \leq\right.$ $\left.\left\langle d_{i}, x\right\rangle \leq m\right\}$ can be represented as (the restriction of) a weighted sum of split functions $\left.\left\{l_{H_{d_{i},},}\right\}\right\}_{k=-m+1}^{m-1}$ and an affine function.

\section{Metrics as Discrete Concave Functions}

By regarding metrics as discrete concave functions and applying the results of Sections 2 and 3, we derive in this section Bandelt-Dress' split decomposition of metrics and some other important concepts of T-theory.

First we briefly review T-theory and the split decomposition of metrics. Let $V$ be a finite set. A function $d: V \times V \rightarrow \mathbf{R}$ is said to be a metric if it satisfies $d(i, i)=0$, $d(i, j)=d(j, i) \geq 0$, and $d(i, j) \leq d(i, k)+d(j, k)$ for $i, j, k \in V$. A polyhedron $P(d) \subseteq \mathbf{R}^{V}$ associated with metric $d$ is defined as

$$
P(d)=\left\{p \in \mathbf{R}^{V} \mid p(i)+p(j) \geq d(i, j)(i, j \in V)\right\} .
$$

The tight span of metric $d$ is a subset of $P(d)$ defined as

$$
T(d)=\left\{p \in \mathbf{R}^{V} \mid \forall i \in V, p(i)=\sup _{j \in V}(d(i, j)-p(j))\right\},
$$

which has been introduced independently by Isbell [17] and Dress [7]. It is known that $T(d)$ coincides with the union of all bounded faces of $P(d)$ [8, Lemma 1].

A split $\{A, B\}$ is a bipartition of $V$, which means that $A \cap B=\emptyset, A \cup B=V$, $A, B \neq \emptyset$. A split metric $\delta_{\{A, B\}}$ associated with a split $\{A, B\}$ is defined as

$$
\delta_{\{A, B\}}(i, j)= \begin{cases}0 & \text { if } \quad i, j \in A \quad \text { or } \quad i, j \in B \quad(i, j \in V) . \\ 1 & \text { otherwise }\end{cases}
$$

For a metric $d$ and a split $\{A, B\}$, the isolation index $\alpha_{\{A, B\}}^{d}$ is the nonnegative number 
defined as

$$
\alpha_{\{A, B\}}^{d}=\frac{1}{2} \min _{i, j \in A, k, l \in B}\left\{\max \left\{\begin{array}{l}
d(i, k)+d(j, l) \\
d(i, l)+d(j, k) \\
d(i, j)+d(k, l)
\end{array}\right\}-d(i, j)-d(k, l)\right\} .
$$

Let $\mathcal{S}(d)$ be the collection of splits defined as

$$
\mathcal{S}(d)=\left\{S: \text { split on } V: \alpha_{S}^{d}>0\right\} .
$$

An additive decomposition of a metric $d=d_{1}+d_{2}$ is said to be coherent if it satisfies $P(d)=P\left(d_{1}\right)+P\left(d_{2}\right)$. A metric $d$ is said to be split-prime if it satisfies $\alpha_{S}^{d}=0$ for each split $S$ The split decomposition theorem is as follows:

Theorem 4.1 [1]. A metric d can be coherently decomposed as

$$
d=\sum_{S \in \mathcal{S}(d)} \alpha_{S}^{d} \delta_{S}+d^{\prime}
$$

where $d^{\prime}$ is a split-prime metric. Moreover, if $d$ is coherently decomposed as

$$
d=\sum_{S \in \mathcal{S}} \lambda_{S} \delta_{S}+d^{\prime \prime}
$$

for a collection of splits $\mathcal{S}$, a positive weight $\lambda \in \mathbf{R}_{++}^{\mathcal{S}}$, and a split-prime metric $d^{\prime \prime}$, then we have $\mathcal{S}=\mathcal{S}(d), \lambda_{S}=\alpha_{S}^{d}$ for $S \in \mathcal{S}$, and $d^{\prime \prime}=d^{\prime}$.

The collection of splits $\mathcal{S}(d)$ is necessarily weakly compatible, that is, for any three splits $S_{1}=\left\{A_{1}, B_{1}\right\}, S_{2}=\left\{A_{2}, B_{2}\right\}$, and $S_{3}=\left\{A_{3}, B_{3}\right\}$ in $\mathcal{S}(d)$, there exist no four points $a, a_{1}, a_{2}, a_{3} \in V$ with $\left\{a, a_{1}, a_{2}, a_{3}\right\} \cap A_{i}=\left\{a, a_{i}\right\}$ for $i=1,2,3$.

As already mentioned in the Introduction, we regard a metric as a function defined on a finite set of points. In the following we deal with a more general object, a distance function, rather than a metric. A distance function on $V$ is a function $d: V \times V \rightarrow \mathbf{R}$ such that $d(i, i)=0$ and $d(i, j)=d(j, i) \geq 0$ for $i, j \in V$, where the triangle inequality is not imposed. For each $A \subseteq V$, we denote by $\chi_{A}$ the characteristic vector of $A$ defined as $\chi_{A}(i)=1$ if $i \in A$ and 0 otherwise. In particular, we write simply $\chi_{i}$ instead of $\chi_{\{i\}}$ for each $i \in V$. Consider the point set

$$
\Lambda=\left\{\chi_{i}+\chi_{j} \mid i, j \in V\right\} .
$$

Then a distance function $d$ is naturally regarded as a function $d: \Lambda \rightarrow \mathbf{R}$ defined by

$$
d\left(\chi_{i}+\chi_{j}\right)=d(i, j) \quad(i, j \in V) .
$$

The following is easily observed.

Lemma 4.2. A function $f: \Lambda \rightarrow \mathbf{R}$ with $f\left(2 \chi_{i}\right)=0$ for $i \in V$ is convex-extensible if and only if it satisfies $f\left(\chi_{i}+\chi_{j}\right) \leq 0$ for $i, j \in V$. 


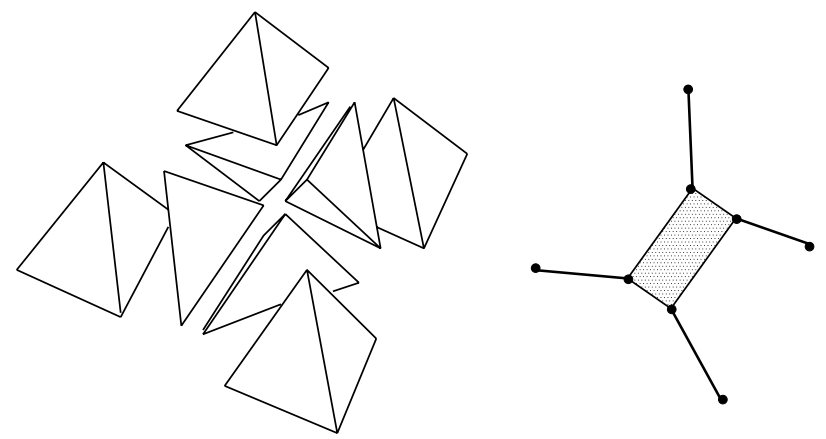

Fig. 6. $\mathcal{T}^{\Lambda}(\overline{(-d)})$ (left) and $T(d)$ (right) of a generic 4-point metric $d$.

Hence it is natural to regard any distance function $d: V \times V \rightarrow \mathbf{R}$ as a discrete concave function on $\Lambda$. Since aff $\Lambda$ coincides with $K=\left\{x \in \mathbf{R}^{V} \mid \sum_{i \in V} x(i)=2\right\}$, we can apply the results of the previous section in a straightforward manner.

The homogeneous convex extension of $-d$ is given by

$$
\begin{aligned}
\overline{(-d)}(x) & =\inf \left\{\sum_{i, j \in V} \lambda_{i j}(-d(i, j)) \mid \sum_{i, j \in V} \lambda_{i j}\left(\chi_{i}+\chi_{j}\right)=x, \lambda_{i j} \geq 0(i, j \in V)\right\} \\
& =\sup \{\langle p, x\rangle \mid p(i)+p(j) \leq-d(i, j)(i, j \in V)\} \\
& =\sup \{\langle p, x\rangle \mid-p \in P(d)\} \quad\left(x \in \mathbf{R}^{V}\right),
\end{aligned}
$$

where for $i, j \in V, i j$ denotes an unordered pair, which means that $i j$ and $j i$ are not distinguished from each other. Hence $\overline{-d}$ is the support function of $-P(d)$. Using the notation (3.2), we have $Q(-d)=-P(d)$. This implies that the tight span $T(d)$ has a dual structure of $\mathcal{T}^{\Lambda}(\overline{-d})$ (recall (N1), (N2), and (N3) in Section 2.3 and see Fig. 6). This duality relation is also suggested by Sturmfels and $\mathrm{Yu}[26]$ in connection with the triangulation of the second hypersimplex $\operatorname{conv}\left\{\chi_{i}+\chi_{j} \mid i, j \in V, i \neq j\right\}$.

Next we derive the split decomposition of metrics (Theorem 4.1) in a more generalized form using the result of the present paper. For this, we begin by establishing a relationship between splits and hyperplanes. For $A, B \subseteq V$ with $A, B \neq \emptyset$ and $A \cap B=\emptyset$, we call the unordered pair $\{A, B\}$ a partial split. A hyperplane $H_{\{A, B\}}$ associated with partial split $\{A, B\}$ is defined as

$$
H_{\{A, B\}}=\left\{x \in \mathbf{R}^{V} \mid x(A)=x(B)\right\},
$$

where we denote $\sum_{i \in A} x(i)$ by $x(A)$ for $x \in \mathbf{R}^{V}$ and $A \subseteq V$.

Lemma 4.3. Let $\{A, B\}$ be a partial split on $V$ and let $C=V \backslash(A \cup B)$. Then we have

$$
\left\{\begin{array}{l}
\Lambda \cap H_{\{A, B\}}=\left\{\chi_{i}+\chi_{j} \mid i \in A, j \in B \text { or } i, j \in C\right\}, \\
\Lambda \cap H_{\{A, B\}}^{+}=\left\{\chi_{i}+\chi_{j} \mid i, j \in A \cup C\right\}, \\
\Lambda \cap H_{\{A, B\}}^{-}=\left\{\chi_{i}+\chi_{j} \mid i, j \in B \cup C\right\}, \\
\Lambda \cap H_{\{A, B\}}^{++}=\left\{\chi_{i}+\chi_{j} \mid i \in A, j \in A \cup C\right\}, \\
\Lambda \cap H_{\{A, B\}}^{-}=\left\{\chi_{i}+\chi_{j} \mid i \in B, j \in A \cup C\right\},
\end{array}\right.
$$

where we regard $H_{\{A, B\}}$ as $H_{\chi_{A}-\chi_{B}, 0}$. 

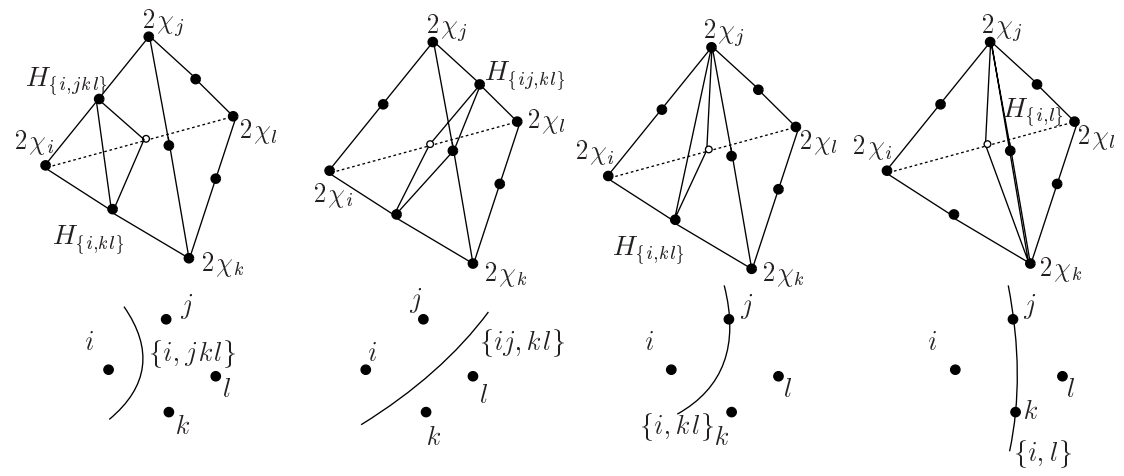

Fig. 7. Examples of the split-hyperplane correspondence.

Figure 7 illustrates small examples of the correspondence between partial splits and hyperplanes. In this figure a partial split and an element of $V$ are represented by a line and a point, respectively. The line corresponding to a partial split $\{A, B\}$ separates points of $A$ and $B$ and contains points of $V \backslash A \cup B$. The family $\mathcal{H}_{\Lambda}$ of linear hyperplanes, as defined in (3.13) together with (a0) and (a1), can be identified in terms of partial splits as follows.

Proposition 4.4. $\mathcal{H}_{\Lambda}=\left\{H_{\{A, B\}} \mid\{A, B\}\right.$ is a partial split on $\left.V\right\}$.

Proof. We show that $H_{A, B}$ satisfies conditions (a0) and (a1). Condition (a0) is clearly satisfied. conv $\Lambda$ is the simplex having edges $\left\{\left[2 \chi_{i}, 2 \chi_{j}\right] \mid i, j \in V\right\}$. By Lemma 4.3, $2 \chi_{i} \in H_{A, B}^{++}$and $2 \chi_{j} \in H_{A, B}^{--}$if and only if $i \in A$ and $j \in B$. Therefore, we have $\left[2 \chi_{i}, 2 \chi_{j}\right] \cap H_{A, B}=\left\{\chi_{i}+\chi_{j}\right\} \in X$ for $i \in A, j \in B$. Conversely, let $H_{a, 0} \in \mathcal{H}_{\Lambda}$. Define $A=\left\{i \in V \mid 2 \chi_{i} \in H_{a, 0}^{++}\right\}$and $B=\left\{i \in V \mid 2 \chi_{i} \in H_{a, 0}^{--}\right\}$. Then $\{A, B\}$ is a partial split on $V$. We show $H_{a, 0}=H_{\{A, B\}}$. By the definition of $\{A, B\}$, we have $a(i)=0$ for $i \in V \backslash(A \cup B)$. For any $2 \chi_{i} \in H_{a, 0}^{++}$and $2 \chi_{i} \in H_{a, 0}^{--}$, by $\Lambda$-admissibility of $\left\{H_{a, 0}\right\}$, it is necessary that $\chi_{i}+\chi_{j} \in H_{a, 0}$ by (a1). Hence we have $a(i)=-a(j)$ for $i \in A, j \in B$. This implies $H_{a, 0}=H_{\{A, B\}}$.

For a partial split $\{A, B\}$, we define a partial split distance $\zeta_{\{A, B\}}$ associated with $\{A, B\}$ as

$$
\zeta_{\{A, B\}}(i, j)= \begin{cases}1 & \text { if } i \in A, j \in B \quad \text { or } \quad i \in B, j \in A \quad(i, j \in V) . \\ 0 & \text { otherwise }\end{cases}
$$

If $A \cup B \neq V$, then $\zeta_{\{A, B\}}$ is not a metric. If $A \cup B=V$, i.e., $\{A, B\}$ is a split, then $\zeta_{\{A, B\}}$ coincides with the split metric $\delta_{\{A, B\}}$.

The following shows that a partial split distance $\zeta_{\{A, B\}}$ associated with partial split $\{A, B\}$ is represented as split function $l_{H_{\{A, B\}}}$ associated with hyperplane $H_{\{A, B\}}$. 
Proposition 4.5. For a partial split $\{A, B\}$, the partial split distance $\zeta_{\{A, B\}}: \Lambda \rightarrow \mathbf{R}$ is represented as

$$
\zeta_{\{A, B\}}(x)=-|x(A)-x(B)| / 2+x(A \cup B) / 2 \quad(x \in \Lambda) .
$$

Moreover, we have

$$
\overline{\left(-\zeta_{\{A, B\}}\right)}(x)=|x(A)-x(B)| / 2-x(A \cup B) / 2+\delta_{\mathbf{R}_{+}^{V}} \quad\left(x \in \mathbf{R}^{V}\right),
$$

where $\delta_{\mathbf{R}_{+}^{V}}$ is the indicator function for $\mathbf{R}_{+}^{V}$; see (2.5).

Proof. Equation (4.14) is obtained by direct calculations. Equation (4.15) follows from $\Lambda$-admissibility of $\left\{H_{\{A, B\}}\right\}$ (Proposition 4.4), Proposition 3.5, and Lemma 3.1(1).

$c_{H_{[A, B\}}}(-d)$ can be explicitly calculated as follows.

Theorem 4.6. For a distance function $d$ and a partial split $\{A, B\}$, we have

$$
c_{H_{\{A, B\}}}(\overline{(-d)})=\max \left\{0, \min \left\{\rho_{A, B}^{d}, \sigma_{A, B}^{d}, \sigma_{B, A}^{d}, \tau_{A, B}^{d}\right\}\right\} \sqrt{|A \cup B|},
$$

where

$$
\begin{aligned}
& \rho_{A, B}^{d}=\frac{1}{2} \min \left\{\begin{array}{l|l}
\max \left\{\begin{array}{l}
d(i, k)+d(j, l) \\
d(i, l)+d(j, k)
\end{array}\right\}-d(i, j)-d(k, l) & \begin{array}{l}
i, j \in A \\
k, l \in B
\end{array}
\end{array},,\right. \\
& \sigma_{A, B}^{d}=\frac{1}{2} \min \left\{\begin{array}{l|l}
d(i, k)+d(i, l)-d(k, l)-2 d(i, j) & \begin{array}{l}
i \in A, k, l \in B \\
j \in V \backslash(A \cup B)
\end{array}
\end{array}\right\}, \\
& \tau_{A, B}^{d}=\min \left\{\begin{array}{l|l}
d(i, k)+d(j, l)-d(i, j)-d(k, l) \mid \begin{array}{c}
i \in A, k \in B \\
j, l \in V \backslash(A \cup B)
\end{array}
\end{array}\right\},
\end{aligned}
$$

and we define $\sigma_{A, B}^{d}=+\infty$ and $\tau_{A, B}^{d}=+\infty$ if $A \cup B=V$.

Proof. We apply formulas (3.14) and (3.15) in Theorem 3.4. Let $C=V \backslash(A \cup B)$. Then $\tilde{c}_{H_{S}}(-d)$ is the minimum of

$$
\frac{-d(i, j)-\overline{(-d)^{\Lambda \cap H_{\{A, B\}}}}(w)}{2 l_{H_{\{A, B\}}}\left(\chi_{i}+\chi_{j}\right)}+\frac{-d(k, l)-\overline{(-d)^{\Lambda \cap H_{\{A, B\}}}}(w)}{2 l_{H_{\{A, B\}}}\left(\chi_{k}+\chi_{l}\right)}
$$

over $i \in A, j \in A \cup C, k \in B$, and $l \in B \cup C$, where $\{w\}=H_{\{A, B\}} \cap\left[\chi_{i}+\chi_{j}, \chi_{k}+\chi_{l}\right]$.

Case 1: $i, j \in A, k, l \in B$. Formula (4.20) is given by

$$
\frac{\sqrt{|A \cup B|}}{2}\left\{-d(i, j)-d(k, l)-2 \overline{(-d)^{\Lambda \cap H_{S}}}\left(\left(\chi_{i}+\chi_{j}+\chi_{k}+\chi_{l}\right) / 2\right)\right\} .
$$

Furthermore, $\overline{(-d)^{\Lambda \cap H_{\{A, B\}}}}\left(\left(\chi_{i}+\chi_{j}+\chi_{k}+\chi_{l}\right) / 2\right)$ is given by the optimal value of linear program

$$
\begin{array}{ll}
\min . & \sum_{u v \in\{i k, i l, j k, j l\}} \lambda_{u v}(-d(u, v)) \\
\text { s.t. } & \sum_{u v \in\{i k, i l, j k, j l\}} \lambda_{u v}\left(\chi_{u}+\chi_{v}\right)=\left(\chi_{i}+\chi_{j}+\chi_{k}+\chi_{l}\right) / 2, \\
& \lambda_{u v} \geq 0 \quad(u v \in\{i k, i l, j k, j l\}) .
\end{array}
$$


By direct calculations, the optimal value of the problem is

$$
\min \{-d(i, k)-d(j, l),-d(i, l)-d(j, k)\} / 2 .
$$

Hence we have

$$
(4.20)=\frac{\sqrt{|A \cup B|}}{2}\left\{-d(i, j)-d(k, l)+\max \left\{\begin{array}{l}
d(i, k)+d(j, l) \\
d(i, l)+d(j, k)
\end{array}\right\}\right\} .
$$

Case 2: $i \in A, j \in C, k, l \in B$. Formula (4.20) is given by

$$
\frac{\sqrt{|A \cup B|}}{2}\left\{-2 d(i, j)-d(k, l)-3 \overline{(-d)^{\Lambda \cap H_{\{A, B\}}}}\left(\left(2 \chi_{i}+2 \chi_{j}+\chi_{k}+\chi_{l}\right) / 3\right)\right\} .
$$

In a similar way to Case 1 , we have

$$
\overline{(-d)^{\Lambda \cap H_{\{A, B\}}}}\left(\left(2 \chi_{i}+2 \chi_{j}+\chi_{k}+\chi_{l}\right) / 3\right)=-(d(i, k)+d(i, l)) / 3 .
$$

Hence we obtain

$$
(4.20)=\frac{\sqrt{|A \cup B|}}{2}\{-2 d(i, j)-d(k, l)+d(i, k)+d(i, l)\} .
$$

Case 3: $i, j \in A, k \in B, l \in C$. It follows from Case 2 by interchanging $A$ and $B$.

Case 4: $i \in A, k \in B, j, l \in C$. Formula (4.20) is given by

$$
\sqrt{|A \cup B|}\left\{-d(i, j)-d(k, l)-2 \overline{2(-d)^{\Lambda \cap H_{\{A, B\}}}}\left(\left(\chi_{i}+\chi_{j}+\chi_{k}+\chi_{l}\right) / 2\right)\right\} .
$$

In a similar way to Case 1 , we have

$$
\overline{(-d)^{\Lambda \cap H_{\{A, B\}}}}\left(\left(\chi_{i}+\chi_{j}+\chi_{k}+\chi_{l}\right) / 2\right)=-(d(i, k)+d(j, l)) / 2 .
$$

Hence, we obtain

$$
(4.20)=\sqrt{|A \cup B|}\{-d(i, j)-d(k, l)+d(i, k)+d(j, l)\} .
$$

Combining (4.21), (4.22), and (4.23), we obtain the desired formula (4.16).

In particular, if a partial split $\{A, B\}$ forms a split, then we have

$$
c_{H_{\{A, B\}}}(\overline{-d})=\alpha_{\{A, B\}}^{d} \sqrt{|V|}
$$

(see the definitions of $\alpha_{\{A, B\}}^{d}$ in (4.4) and $\rho_{A, B}^{d}$ in (4.17)). Accordingly, the isolation index can be extended for a partial split $\{A, B\}$ by defining

$$
\alpha_{\{A, B\}}^{d}=\max \left\{0, \min \left\{\rho_{A, B}^{d}, \sigma_{A, B}^{d}, \sigma_{B, A}^{d}, \tau_{A, B}^{d}\right\}\right\} .
$$

Similarly, $\mathcal{S}(d)$ can be extended for partial splits by defining

$$
\mathcal{S}(d)=\left\{S: \text { partial split on } V: \alpha_{S}^{d}>0\right\} .
$$

A distance $d$ is said to be split-prime if $\alpha_{S}^{d}=0$ for each partial split $S$. An additive decomposition of a distance $d=d_{1}+d_{2}$ is said to be coherent if $P(d)=P\left(d_{1}\right)+P\left(d_{2}\right)$. Note that this condition is equivalent to $\overline{(-d)}=\overline{\left(-d_{1}\right)}+\overline{\left(-d_{2}\right)}$.

As a consequence of above arguments, we obtain an extension of Bandelt-Dress' split decomposition (Theorem 4.1). 
Theorem 4.7. A distance function $d: V \times V \rightarrow \mathbf{R}$ can be coherently decomposed as

$$
d=\sum_{S \in \mathcal{S}(d)} \alpha_{S}^{d} \zeta_{S}+d^{\prime}
$$

where $d^{\prime}$ is a split-prime distance. Moreover, if $d$ is coherently decomposed as

$$
d=\sum_{S \in \mathcal{S}} \lambda_{S} \zeta_{S}+d^{\prime \prime}
$$

for a collection of partial splits $\mathcal{S}$, a positive weight $\lambda \in \mathbf{R}_{++}^{\mathcal{S}}$ and a split-prime distance $d^{\prime \prime}$, then we have $\mathcal{S}=\mathcal{S}(d), \lambda_{S}=\alpha_{S}^{d}$ for $S \in \mathcal{S}(d)$, and $d^{\prime \prime}=d^{\prime}$.

Proof. Decompose $-d$ into the form (3.4) in Theorem 3.2 and apply Propositions 4.4 and 4.5, Theorem 4.6 and Lemma 4.2. Then we obtain (4.27). The coherency of the decomposition (4.27) follows from $Q(-d)=-P(d)$. We show the latter part by $\sum_{S \in \mathcal{S}(d)} \alpha_{S}^{d \overline{\left(-\zeta_{S}\right)}}+\overline{\left(-d^{\prime}\right)}=\sum_{S \in \mathcal{S}} \lambda_{S} \overline{\left(-\zeta_{S}\right)}+\overline{\left(-d^{\prime \prime}\right)}$, Proposition 4.5, and Theorem 2.2. We have $\mathcal{S}(d)=\mathcal{S}, \alpha_{S}^{d}=\lambda_{S}$, and $\overline{\left(-d^{\prime}\right)}=\overline{\left(-d^{\prime \prime}\right)}$. By the convex-extensibility of distances (Lemma 4.2), we have $-d^{\prime}={\overline{\left(-d^{\prime}\right)}}^{\Lambda}={\overline{\left(-d^{\prime \prime}\right)}}^{\Lambda}=-d^{\prime \prime}$.

We call (4.27) the extended split decomposition of $d$. The cardinality of $\mathcal{S}(d)$ is bounded by $|V|(|V|-1) / 2$ by Proposition 3.6. Therefore the decomposition (4.27) is also obtained in polynomial time by an algorithm similar to that for the split decomposition of a metric [1]. It is easily observed that if $d$ satisfies the triangle inequality, $\tau_{\{A, B\}}^{d} \leq 0$ holds for any proper partial split $\{A, B\}$ with $A \cup B \neq V$. This implies that $\mathcal{S}(d)$ consists of splits. Hence if $d$ is a metric, the decomposition (4.27) coincides with the split decomposition of metrics (4.6).

Remark 4.8. Any symmetric function $f: V \times V \rightarrow \mathbf{R}$ with $f(i, j)=f(j, i)$ for $i, j \in V$ can be regarded as $f: \Lambda \rightarrow \mathbf{R}$ by the correspondence (4.9). Similarly, $P(f)$ and $T(f)$ are also definable. The arguments in this section can be adapted to any symmetric function on $V$. In fact, Bandelt and Dress [1] discuss the split decomposition (4.6) for symmetric functions. However, they [1] use only split metrics in the decomposition (4.6). In the case that $d$ violates triangle inequality, it is possible to achieve a more precise decomposition of $d$ by (4.27) since (4.27) uses also partial split distances. For example, consider the following 4-point distance function:

$$
d=\begin{array}{|l|llll|}
\hline & i & j & k & l \\
\hline i & 0 & 3 & 5 & 1 \\
j & 3 & 0 & 1 & 5 \\
k & 5 & 1 & 0 & 2 \\
l & 1 & 5 & 2 & 0 \\
\hline
\end{array} .
$$

Note that $d$ violates the triangle inequality. By the extended split decomposition (4.27), 


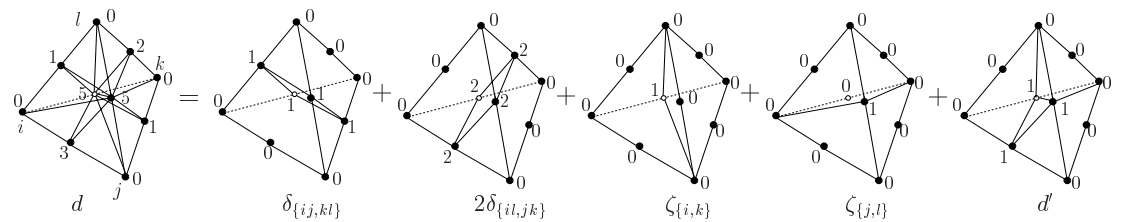

Fig. 8. An example of the extended split decomposition.

$d$ is decomposed as

$$
d=\delta_{\{i j, k l\}}+2 \delta_{\{i k, j l\}}+\zeta_{\{i, k\}}+\zeta_{\{j, l\}}+\begin{array}{|c|cccc}
\hline & i & j & k & l \\
\hline i & 0 & 1 & 1 & 0 \\
j & 1 & 0 & 0 & 1 \\
k & 1 & 0 & 0 & 0 \\
l & 0 & 1 & 0 & 0 \\
\hline
\end{array}
$$

In the ordinary Bandelt-Dress' split decomposition (4.6), only the first two term of the right-hand side of (4.30) are exploited and the sum of the last three terms is treated as the split-prime residue. Figure 8 illustrates this decomposition in term of the subdivision $\mathcal{T}^{\Lambda}(\overline{(-d)})$. This indicates that the extended split decomposition exploits more detailed combinatorial structures of distances than Bandelt-Dress' split decomposition.

Remark 4.9. It is well known that every 4-point metric is totally split-decomposable, i.e., a split-prime residue $d^{\prime}$ of (4.6) vanishes. It is easily seen that every 3-point distance function is also totally split-decomposable in our sense, i.e., $d^{\prime}=0$ in the decomposition (4.27). However, as is seen in Remark 4.8, not every 4-point distance function is totally split-decomposable in our sense.

Remark 4.10. By the decomposition (4.27), $P(d)$ is decomposed as

$$
P(d)=Z(d)+P\left(d^{\prime}\right),
$$

where $Z(d)$ is (a translation of) the maximum zonotopic summand given as

$$
Z(d)=\sum_{\{A, B\} \in \mathcal{S}(d)} \alpha_{\{A, B\}}^{d}\left(\left[\chi_{A}-\chi_{B}, \chi_{B}-\chi_{A}\right] / 2+\chi_{A \cup B} / 2\right) .
$$

If $d^{\prime}=0$, we have $P(d)=Z(d)+\mathbf{R}_{+}^{V}$. In this case, tight span $T(d)$ is the union of the faces of $Z(d)$ whose normal cone contains negative vectors.

Remark 4.11. The coherent decomposition decomposes a distance $d$ into a sum of distances which induce a coarser subdivision than $\mathcal{T}^{\Lambda}(\overline{-d})$. In particular, it is observed from the subdivision of conv $\left\{2 \chi_{i}, 2 \chi_{j}, 2 \chi_{k}\right\}$ induced by $\mathcal{T}^{\Lambda}(\overline{(-d)})$ that $d$ satisfies $d(i, j) \leq d(i, k)+d(j, k)$ if and only if $\operatorname{conv}\left\{2 \chi_{k}, \chi_{i}+\chi_{j}\right\} \notin \mathcal{T}^{\Lambda}(\overline{(-d)})$ (see Fig. 9). This implies that in any coherent decomposition $d=d_{1}+d_{2}$, if $d$ is a metric, then both $d_{1}$ and $d_{2}$ are necessarily metrics. 


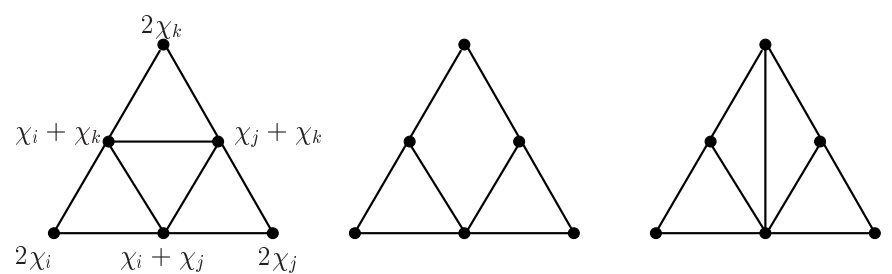

Fig. 9. The role of triangle inequality: $d(i, j)<d(i, k)+d(j, k)$ (left), $d(i, j)=d(i, k)+d(j, k)$ (center), and $d(i, j)>d(i, k)+d(j, k)$ (right).

Remark 4.12. Koolen et al. [18] introduced the coherency index as a direct generalization of the isolation index. For two metrics $d^{\prime}$ and $d$, the coherency index is the nonnegative value $\alpha_{d^{\prime}}^{d}$ which has the property that $d=\alpha d^{\prime}+\left(d-\alpha d^{\prime}\right)$ is a coherent decomposition if and only if $0 \leq \alpha \leq \alpha_{d^{\prime}}^{d}\left[18\right.$, Theorem 4.1]. By definition, $\alpha_{\zeta_{S}}^{d}=\alpha_{S}^{d}$ must hold for a partial split $S$. They give a formula of $\alpha_{d^{\prime}}^{d}$ as

$$
\alpha_{d^{\prime}}^{d}=\min _{f: \text { vertex of } P(d)} \max _{f^{\prime}: \text { vertex of } P\left(d^{\prime}\right)} m\left(f, f^{\prime}\right),
$$

where $m\left(f, f^{\prime}\right)$ is defined as

$$
m\left(f, f^{\prime}\right)=\min _{i, j \in V: f^{\prime}(i)+f^{\prime}(j) \neq d^{\prime}(i, j)} \frac{f(i)+f(j)-d(i, j)}{f^{\prime}(i)+f^{\prime}(j)-d^{\prime}(i, j)} .
$$

This formula comes from the fact that $P(d)=\alpha P\left(d^{\prime}\right)+P\left(d-\alpha d^{\prime}\right)$ if and only if each $f \in P(d)$ and $f^{\prime} \in P\left(d^{\prime}\right)$ satisfies $f-\alpha f^{\prime} \in P\left(d-\alpha d^{\prime}\right)$. It seems to be difficult to derive the explicit formula (4.25) of $\alpha_{\zeta_{S}}^{d}=\alpha_{S}^{d}$ from the coherency index (4.33). It should be noted that our derivation of $\alpha_{S}^{d}$ comes from the fact that $P(d)=\alpha P\left(d^{\prime}\right)+P\left(d-\alpha d^{\prime}\right)$ if and only if $\overline{(-d)}-\alpha \overline{\left(-d^{\prime}\right)}$ is convex on $\mathbf{R}_{+}^{V}$.

Next we characterize the $\Lambda$-admissibility of hyperplanes in terms of combinatorial properties of the corresponding partial splits. First we observe that $\mathcal{S}(d)$ does not contain the following types of partial splits (see Fig. 10):

(C1) Three partial splits $\left\{A_{1}, B_{1}\right\},\left\{A_{2}, B_{2}\right\},\left\{A_{3}, B_{3}\right\}$ and four points $a, a_{1}, a_{2}, a_{3} \in$ $V$ such that $\left\{a, a_{1}, a_{2}, a_{3}\right\} \cap A_{i}=\left\{a, a_{i}\right\}$ for $i=1,2,3$ (the violation of weak compatibility).

(C2) Two partial splits $\left\{A_{1}, B_{1}\right\},\left\{A_{2}, B_{2}\right\}$ and three points $a, b, c \in V$ such that $a \in A_{1}, b, c \in B_{1}, b \in A_{2}, c \in B_{2}$, and $a \in V \backslash\left(A_{2} \cup B_{2}\right)$.

(C3) Two partial splits $\left\{A_{1}, B_{1}\right\},\left\{A_{2}, B_{2}\right\}$ and three points $a, b, c \in V$ such that $b \in A_{1}, c \in B_{1}, a \in V \backslash\left(A_{1} \cup B_{1}\right), a \in A_{2}, b \in B_{2}$, and $c \in V \backslash\left(A_{2} \cup B_{2}\right)$.

For (C1), observe that not all three $\rho_{A_{1}, B_{1}}^{d}, \rho_{A_{2}, B_{2}}^{d}$ and $\rho_{A_{3}, B_{3}}^{d}$ are positive. Similarly, for (C2), observe that both $\rho_{A_{1}, B_{1}}^{d}$ and $\tau_{A_{2}, B_{2}}^{d}$ are not positive. To see (C3), observe that both $\tau_{A_{1}, B_{1}}^{d}$ and $\tau_{A_{2}, B_{2}}^{d}$ are not positive. Of course, these can be geometrically checked by the corresponding hyperplanes in Fig. 10; in each case there is a vertex, indicated by the arrow, not contained by $\Lambda$. 

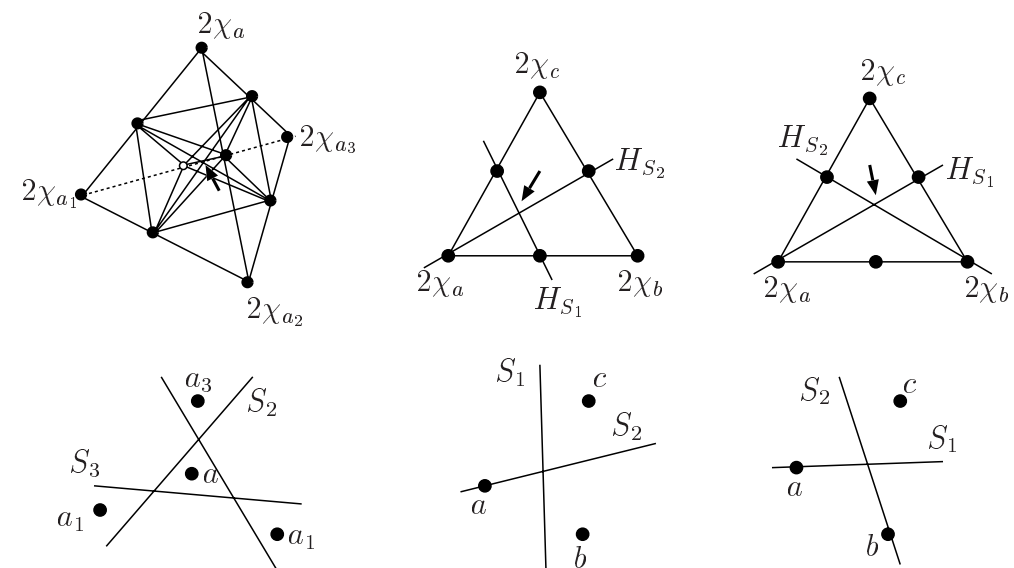

$(\mathrm{C} 1)^{S}$

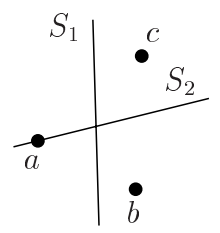

$(\mathrm{C} 2)$

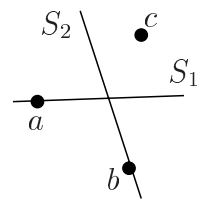

(C3)

Fig. 10. Forbidden partial splits and the corresponding hyperplanes.

A collection of partial splits free from $(\mathrm{C} 1)-(\mathrm{C} 3)$ is said to be weakly compatible, which is clearly an extension of the weak compatibility of splits. The following is an extension of Theorem 3 of [1].

Theorem 4.13. Let $\mathcal{S}$ be a weakly compatible collection of partial splits on $V$. For $\lambda \in \mathbf{R}_{++}^{\mathcal{S}}$, let a distance function $d: V \times V \rightarrow \mathbf{R}$ be defined as $d=\sum_{S \in \mathcal{S}} \lambda_{S} \zeta_{S}$. Then we have $\mathcal{S}=\mathcal{S}(d)$ and $\lambda_{S}=\alpha_{S}^{d}$ for each $S \in \mathcal{S}$.

Proof. We adapt the proof of Theorem 3 of [1]. For a partial split $\{A, B\}$, let $\tilde{\alpha}_{\{A, B\}}^{d}$ be defined as

$$
\tilde{\alpha}_{\{A, B\}}^{d}=\min \left\{\rho_{A, B}^{d}, \sigma_{A, B}^{d}, \sigma_{B, A}^{d}, \tau_{A, B}^{d}\right\}
$$

It suffices to show

$$
\tilde{\alpha}_{S}^{d} \geq \lambda_{S} \quad(S \in \mathcal{S}) .
$$

By the formula of $\alpha_{S}^{d}$ for a partial split $S=\{A, B\}$, there exists $Y \subseteq V$ with $A \cap Y \neq \emptyset$, $B \cap Y \neq \emptyset$, and $|Y| \leq 4$ such that

$$
\tilde{\alpha}_{\{A, B\}}^{d}=\tilde{\alpha}_{\{A \cap Y, B \cap Y\}}^{d_{Y}},
$$

where $d_{Y}: Y \times Y \rightarrow \mathbf{R}$ denotes the restriction of $d$ to $Y$. Hence it is sufficient to show (4.35) for the case $|V| \leq 4$.

In the case of $|V|=2$, (4.35) is obvious. In the case of $|V|=3$, let $V=\{i, j, k\}$. For simplicity of notation, we denote a partial split $\{\{i, j\},\{k\}\}$ by $\{i j, k\}$. It suffices to check (4.35) for $S=\{i j, k\}$, and $S=\{i, j\}$. For $S=\{i j, k\}, \mathcal{S}$ does not contain $\{i, j\}$ by (C2). Hence we have $\tilde{\alpha}_{\{i j, k\}}^{d}=\rho_{i j, k}^{d}=\min \{(d(i, k)+d(j, k)-d(i, j)) / 2, d(i, k), d(j, k)\} \geq$ $\lambda_{\{i j, k\}}$. For $S=\{i, j\}, \mathcal{S}$ does not contain $\{j, k\},\{i, k\}$, and $\{i j, k\}$ from (C2) and (C3). 
We have $\tau_{i, j}^{d}=d(i, j)-d(i, k)-d(j, k)=\lambda_{\{i, j\}}, \rho_{i, j}^{d}=d(i, j) \geq \lambda_{\{i, j\}}, \sigma_{i, j}^{d} \geq \tau_{i, j}^{d}$, and $\sigma_{j, i}^{d} \geq \tau_{i, j}^{d}$. Hence we obtain $\tilde{\alpha}_{\{i, j\}}^{d} \geq \lambda_{\{i, j\}}$. Therefore (4.35) holds for $|V|=3$.

In the case of $|V|=4$, let $V=\{i, j, k, l\}$. We may assume that the minimum of $\tilde{\alpha}_{S}^{d}$ is attained by all different four points (otherwise it can be reduced to the case $|V| \leq 3$ ). It suffices to check (4.35) for $S=\{i j, k l\}, S=\{i j, k\}$ and $S=\{i, j\}$. For $S=\{i j, k l\}$, we have

$$
\tilde{\alpha}_{\{i j, k l\}}^{d}=\rho_{i j, k l}^{d}=\{\max \{d(i, k)+d(j, l), d(i, l)+d(j, k)\}-d(i, j)-d(k, l)\} / 2 .
$$

From condition (C1), $\mathcal{S}$ does not contain both $\{i k, j l\}$ and $\{i l, j k\}$ simultaneously. Suppose that $\{i k, j l\} \in \mathcal{S}$ and $\{i l, j k\} \notin \mathcal{S}$. By conditions (C2) and (C3), the possible partial splits in $\mathcal{S}$ that contribute to the term $d(i, j)+d(k, l)$ are only five partial splits $\{i, j k l\},\{j, i k l\},\{k, i j l\},\{l, i j k\}$, and $\{i k, j l\}$. The first four partial splits contribute equally to $d(i, k)+d(j, l), d(i, l)+d(j, k)$ and $d(i, j)+d(k, l)$. Hence we have $\tilde{\alpha}_{\{i j, k l\}}^{d} \geq \max \left\{\lambda_{\{i j, k l\}}, \lambda_{\{i j, k l\}}+\lambda_{\{i k, j l\}}\right\}-\lambda_{\{i k, j l\}}=\lambda_{\{i j, k l\}}$. For $S=\{i j, k\}$, we have

$$
\tilde{\alpha}_{\{i j, k\}}^{d}=\sigma_{k, i j}^{d}=\{d(i, k)+d(j, k)-d(i, j)-2 d(k, l)\} / 2 .
$$

From conditions (C2) and (C3), the possible partial splits in $\mathcal{S}$ that contribute to the term $d(i, j)+2 d(k, l)$ are four partial splits $\{i, j k l\},\{j, i k l\},\{k, i j l\}$, and $\{i k, j\}$. However, it is easily examined that these partial split distances cancel out in (4.37). Therefore we obtain $\tilde{\alpha}_{\{i j, k\}}^{d} \geq \lambda_{\{i j, k\}}$. For $S=\{i, j\}$, we may assume

$$
\tilde{\alpha}_{\{i, j\}}^{d}=\tau_{i, j}^{d}=d(i, j)+d(k, l)-d(i, k)-d(j, l) .
$$

From conditions (C2) and (C3), the possible partial splits in $\mathcal{S}$ that contribute to the term $d(i, k)+d(j, l)$ are five partial splits $\{i, j k l\},\{j, i k l\},\{i, j k\},\{j, i l\}$, and $\{i l, j k\}$. However, these partial split distances cancel out in (4.38). Therefore we obtain $\tilde{\alpha}_{\{i, j\}}^{d} \geq$ $\lambda_{\{i, j\}}$. Hence we conclude that (4.35) holds for $|V|=4$.

By Theorem 4.13 and Proposition 3.5, the weak compatibility of a collection of splits is also characterized by the $\Lambda$-admissibility of the corresponding collection of hyperplanes.

Theorem 4.14. For a collection of partial splits $\mathcal{S}$, the following conditions are equivalent:

(a) $\mathcal{S}$ is weakly compatible.

(b) $\left\{H_{S} \mid S \in \mathcal{S}\right\}$ is $\Lambda$-admissible.

Figure 11 illustrates all types of maximal weakly compatible partial splits on a 4-point set and the corresponding hyperplane arrangements.

Remark 4.15. The fundamental fact [1, Corollary 4] that the number of weakly compatible splits is bounded by $|V|(|V|-1) / 2$ also follows from Proposition 3.6. It is shown that this bound is attained by the maximum circular split system, which is obtained from a convex $|V|$-gon [1, Theorem 5]. By Corollary 3.7, the sum of maximum circular split 


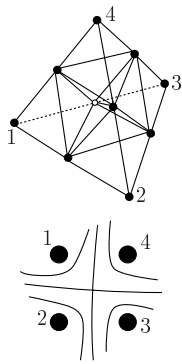

(a)
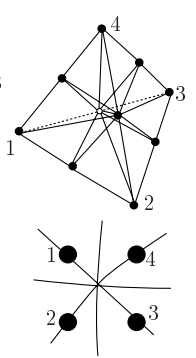

(b)
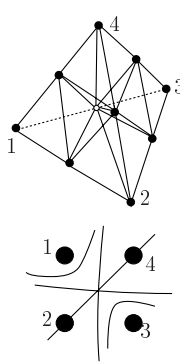

(c)

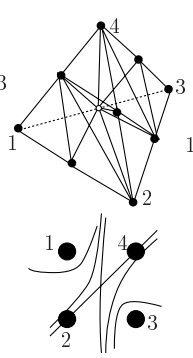

(d)

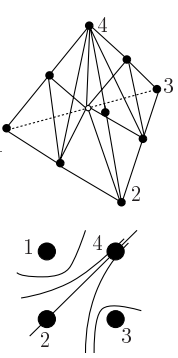

(e)
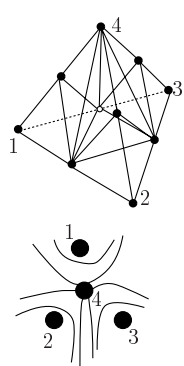

(f)

Fig. 11. Maximal weakly compatible partial splits on a 4-point set and the corresponding hyperplanes.

metrics yields a triangulation of conv $\Lambda$. We point out that this construction of a triangulation of conv $\Lambda$ is essentially equivalent to the construction of the triangulation of the second hypersimplex conv $\left\{\chi_{i}+\chi_{j} \mid i, j \in V, i \neq j\right\}$ due to De Loera et al. [6] (see also Chapter 9 of [25]).

A collection of splits $\mathcal{S}$ is said to be compatible if for any pair of splits $\{A, B\},\{C, D\} \in$ $\mathcal{S}$, at least one of four sets $A \cap C, A \cap D, B \cap C$ and $B \cap D$ is empty (see [5], [2], and [24]). Compatibility of a collection of splits can also be captured as a geometric property of the corresponding collection of hyperplanes.

Proposition 4.16. For a collection of splits $\mathcal{S}$, the following conditions are equivalent:

(a) $\mathcal{S}$ is compatible.

(b) $H_{1} \cap H_{2} \cap$ ri conv $\Lambda=\emptyset$ holds for each pair $H_{1}, H_{2} \in\left\{H_{S} \mid S \in \mathcal{S}\right\}$.

Proof. For two splits $\{A, B\},\{C, D\} \in \mathcal{S}$, consider two hyperplanes $H_{\{A, B\}}$ and $H_{\{C, D\}}$. The nonemptiness of $H_{\{A, B\}} \cap H_{\{C, D\}} \cap$ ri conv $\Lambda$ is equivalent to the existence of a solution to a linear equality, inequality system

$$
\left\{\begin{array}{rl}
x(A)-x(B) & =0, \\
x(C)-x(D) & =0, \\
x(i) & >0
\end{array} \quad(i \in V) .\right.
$$

(a) $\Rightarrow$ (b) By the compatibility of splits $\mathcal{S}$, we may assume $A \subset C$ and $D \subset B$. By subtracting the second of (4.39) from the first, we have $x(B \cap C)=0$ and hence (4.39) is empty. (b) $\Rightarrow$ (a) Suppose that $\{A, B\}$ and $\{C, D\}$ are incompatible, i.e., all four sets $A \cap C, A \cap D, B \cap C$, and $B \cap D$ are nonempty. Then $x \in \mathbf{R}^{V}$ defined by

$$
x(i)= \begin{cases}1 /|A \cap C| & \text { if } \quad i \in A \cap C, \\ 1 /|A \cap D| & \text { if } i \in A \cap D, \\ 1 /|B \cap C| & \text { if } i \in B \cap C, \\ 1 /|B \cap D| & \text { if } i \in B \cap D\end{cases}
$$

is a solution to (4.39). 
A metric $d$ is a tree metric if it is represented as the path metric of some weighted tree. It is well known that $d$ is a tree metric if and only if it is represented as

$$
d=\sum_{S \in \mathcal{S}} \alpha_{S} \delta_{S}
$$

for some compatible collection of splits $\mathcal{S}$ and a positive weight $\alpha \in \mathbf{R}_{++}^{\mathcal{S}}$ (see [5], [2], and [24]). From Propositions 2.9 and 4.16, one of the central theorems in T-theory can be derived.

Theorem 4.17 [7]. A metric d is a tree metric if and only if $T(d)$ is a tree.

Remark 4.18. It is natural to ask when $T(d)$ is a tree for a distance $d$; Theorem 4.17 is the case that $d$ is a metric. This question was answered by Hirai [15], who shows that the following conditions for a distance $d: V \times V \rightarrow \mathbf{R}$ are equivalent:

(1) $T(d)$ is a tree.

(2) $d$ can be represented as $d=\sum_{S \in \mathcal{S}} \alpha_{S} \zeta_{S}$ for a compatible collection of partial splits $\mathcal{S}$ and a positive weight $\alpha \in \mathbf{R}_{++}^{\mathcal{S}}$, where a collection of partial splits $\mathcal{S}$ is said to be compatible if each pair $\{A, B\},\{C, D\} \in \mathcal{S}$ satisfies one of the following four conditions:

$$
\begin{aligned}
& A \subseteq C \text { and } B \supseteq D, \\
& A \subseteq D \text { and } B \supseteq C, \\
& A \supseteq C \text { and } B \subseteq D, \\
& A \supseteq D \text { and } B \subseteq C .
\end{aligned}
$$

(3) There exist some weighted tree $T$ and some collection of subtrees $\left\{T_{i} \mid i \in V\right\}$ of $T$ such that

$$
d(i, j)=\text { the shortest path length between } T_{i} \text { and } T_{j} \text { in } T \quad(i, j \in V)
$$

(see Fig. 3 in the Introduction).

Proposition 4.16 can also be extended for a compatible collection of partial splits. In particular, (e) and (f) in Fig. 11 are such collections of partial splits. Clearly, a compatible partial splits is weakly compatible. So a distance between subtrees of a tree is totally split-decomposable in our sense and therefore can be recognized by the extended split decomposition.

\section{Acknowledgements}

The author thanks Kazuo Murota for helpful comments, Bernd Sturmfels for an insightful suggestion (Remark 3.8), and the anonymous referee for suggestive comments.

\section{References}

1. H.-J. Bandelt and A. W. M. Dress, A canonical decomposition theory for metrics on a finite set, Adv. Math. 92 (1992), 47-105. 
2. J.-P. Barthélémy and A. Guénoche, Trees and Proximity Representations (translated from the French by G. Lawden), Wiley, Chichester, 1991.

3. L. Billera, P. Filliman, and B. Sturmfels, Constructions and complexity of secondary polytopes, Adv. Math. 83 (1990), 155-179.

4. E. D. Bolker, A class of convex bodies, Trans. Amer. Math. Soc. 145 (1969), 323-345

5. P. Buneman, The recovery of trees from measures of dissimilarity, in Mathematics in the Archaeological and Historical Sciences (F. R. Hodson, D. G. Kendall, and P. Tautu, eds.), Edinburgh University Press, Edinburgh, 1971, pp. 387-395.

6. J. A. De Loera, B. Sturmfels, and R. R. Thomas, Gröbner bases and triangulations of the second hypersimplex, Combinatorica 15 (1995), 409-424.

7. A. W. M. Dress, Trees, tight extensions of metric spaces, and the cohomological dimension of certain groups: a note on combinatorial properties of metric spaces, Adv. Math. 53 (1984), 321-402.

8. A. W. M. Dress, Towards a classification of transitive group actions on finite metric spaces, Adv. Math. 74 (1989), 163-189.

9. A. Dress, V. Moulton, and W. Terhalle, T-theory: an overview, European J. Combin. 17 (1996), 161-175.

10. R. M. Erdahl and S. S. Ryshkov, On lattice dicing, European J. Combin. 15 (1994), 459-481

11. A. Frank, An algorithm for submodular functions on graphs, Ann. Discrete Math. 16 (1982), 97-120.

12. S. Fujishige, Theory of submodular programs: a Fenchel-type min-max theorem and subgradients of submodular functions, Math. Program. 29 (1984), 142-155.

13. S. Fujishige, Submodular Functions and Optimization, 2nd edn., Elsevier, Amsterdam, 2005.

14. I. M. Gel'fand, M. M. Kapranov, and A. V. Zelevinsky, Discriminants, Resultants, and Multidimensional Determinants, Birkhäuser, Boston, MA, 1994.

15. H. Hirai, Characterization of a distance between subtrees of a tree associated with tight span, Ann. Combin. 10 (2006), 111-128.

16. H. Hirai and K. Murota, M-convex functions and tree metrics, Japan J. Indust. Appl. Math. 21 (2004), 391-403.

17. J. R. Isbell, Six theorems about injective metric spaces, Comment. Math. Helv. 39 (1964), 65-76.

18. J. Koolen, V. Moulton, and U. Tönges, The coherency index, Discrete Math. 192 (1998), 205-222.

19. L. Lovász, Submodular functions and convexity, in Mathematical Programming-The State of the Art (A. Bachem, M. Grötschel, and B. Korte, eds.), Springer-Verlag, Berlin, 1983, pp. 235-257.

20. K. Murota, Discrete Convex Analysis, SIAM, Philadelphia, PA, 2003.

21. R. T. Rockafellar, Convex Analysis, Princeton University Press, Princeton, NJ, 1970.

22. R. Schneider, Convex Bodies: The Brunn-Minkowski Theory, Cambridge University Press, Cambridge, 1993.

23. A. Schrijver, Theory of Linear and Integer Programming, Wiley, New York, 1986.

24. C. Semple and M. Steel, Phylogenetics, Oxford University Press, Oxford, 2003.

25. B. Sturmfels, Gröbner Bases and Convex Polytopes, American Mathematical Society, Providence, RI, 1996.

26. B. Sturmfels and J. Yu, Classification of six-point metrics, Electron. J. Combin. 11 (2004), R44.

27. G. M. Ziegler, Lectures on Polytopes, Springer-Verlag, New York, 1995.

Received June 25, 2004, and in revised form December 1, 2005, and January 26, 2006.

Online publication June 5, 2006. 